Sharif University of Technology
Scientia Iranica
SCIENTIA
I RAN ICA

\title{
The effect of radial force on pull-in instability and frequency of rigid core circular and annular plates subjected to electrostatic field
}

\author{
M. Khorshidi Paji, M. Dardel*, M.H. Pashaei, and R. Akbari Alashti \\ Department of Mechanical Engineering, Babol Noshirvani University of Technology, Shariati Street, Babol, P.O. Box 484, Postal \\ Code: 47148-71167, Mazandaran, Iran.
}

Received 22 August 2016; received in revised form 20 February 2017; accepted 17 June 2017

\author{
KEYWORDS \\ Pull-in instability; \\ Circular and annular \\ plate; \\ Rigid core; \\ Radial load; \\ Arc length \\ continuation; \\ Natural frequency.
}

\begin{abstract}
This study investigates static pull-in instability and frequency analysis of circular and annular plates in electrical field. The plate is modelled based on the classical plate theory with nonlinear Von Kármán strain-displacement field. The governing equation of motion and boundary conditions were obtained using the Hamilton principle. For this purpose, potential and kinetic energies and the results achieved through radial and electrostatic forces were obtained. Governing partial differential equations were reduced to ordinary differential equations by Galerkin's method. Then, static pull-in instabilities of clamped circular and annular plates with clamped-clamped and clamped-simply supported boundary conditions were analyzed by the arc-length continuation method. The effects of rigid core, radial load, geometric nonlinearity, inner radius, and boundary conditions on pull-in instability and frequency of the plate were studied.
\end{abstract}

(C) 2018 Sharif University of Technology. All rights reserved.

\section{Introduction}

Micro-Electro-Mechanical Systems (MEMS) consist of micro-sized mechanical and electrical elements integrated into a common silicon substrate. Due to high frequency, lightweight, small-sized, and low-energy consumption, MEMS are used widely in many fields. Different types of actuation, such as thermal, magnetic, piezoelectric, and electrostatic actuation, are employed in MEMS. Nowadays, electrostatically actuated MEMS play an important role in many industrial devices such as sensors, microphones, micro-actuators, and micro-pumps. One of the most important problems

\footnotetext{
*. Corresponding author.

E-mail addresses: m.khorshidi.mech@gmail.com (M. Khorshidi Paji); dardel@nit.ac.ir (M. Dardel);

mpashaei@nit.ac.ir (M.H. Pashaei); raalashti@nit.ac.ir (R. Akbari Alashti).
}

doi: $10.24200 /$ sci. 2017.4208 of these systems is nonlinear pull-in instability due to the presence of electrostatic force, causing failure of devices. Thus, the study and prediction of such instability is necessary to the design of such systems.

Pull-in instability was observed experimentally by Nathanson et al. [1] and Taylor [2]. This instability appears when the electrostatic force goes beyond the elastic restoring force of the structure; hence, the substrate is touched [3]. The critical value of voltage in which this instability occurs is represented as the pull-in voltage. If the rate of voltage variation is very low and negligible, inertia has nearly no effect on the microstructure's behavior; accordingly, the pull-in voltage is called the static pull-in voltage. However, when the rate of voltage variation is considerable, the effect of inertia has to be included. The pullin instability related to this situation is called the dynamic pull-in instability; the critical value of voltage, corresponding to the dynamic instability, is referred to as the dynamic pull-in voltage [4]. 
Static pull-in behaviors of different structures of MEMS is studied. In some studies, lumped models, such as one-Degree-Of-Freedom (DOF) mass spring system model $[5,6]$, are used. There are some studies on two DOFs lumped models [6-8]. In addition, there are some studies on pull-in instability of microbeams [9-15]. Baghani et al. [16] studied the vibration of electrostatically actuated double-clamped and simply-supported micro-beams using Variational Iteration Method (VIM). Rahaeifard and Ahmadian [17] investigated the static deflection and pull-in instability of electrostatically actuated micro cantilevers based on the strain gradient theory. Gholami et al. [18] studied the nonlinear pull-in instability of electrostatically actuated micro-switches based on Mindlin's strain gradient elasticity and the Timoshenko beam theory. They solved the problem using the Generalized Differential Quadrature (GDQ) method and the pseudo arc-length continuation technique. Xiao et al. [19] developed a size-dependent model for electrostatically actuated micro-beam with piezoelectric layers attached based on a modified couple stress theory.

However, there are limited studies on membrane and plate structures. There are some studies on pullin instability of rectangular plates, some of which are described here. Srinivas [20] employed the classical linear plate theory to study the static pull-in instability of simply-supported rectangular plates. Mukherjee et al. [21] investigated the pull-in instability of cantilever plate using Finite-Element Method (FEM). Zhao et al. [22] presented a reduced-order model of electrically actuated square micro-plate. Their model accounts for the electric-force nonlinearity and the mid-plane stretching of the plate. They found linear un-damped vibration modes numerically, using the hierarchical FEM, which are applicable to a Galerkin approximation. Moghimi Zand et al. [23] developed a hybrid Finite-Element Method (FEM) and Finite-Difference Method (FDM) to investigate contact phenomenon in micro-plates actuated by ramp voltages. They utilized the hybrid FEM-FDM model to compute values of contact time and dynamic behavior of rectangular multi-layer micro-plates. Wang et al. [24] investigated the effects of surface energy on the pull-in instability of electrostatically actuated rectangular micro/nanoplates based on the modified couple stress theory.

Annular and circular plates have been receiving increasing interest in MEMS community, especially in micro-pump applications [25]. Some researchers have studied pull-in instability of circular plates. Wang et al. [26] investigated the pull-in instability and vibration of a pre-stressed circular electrostatically actuated micro-plate, with consideration of the Casimir force. They used von Kármán's nonlinear bending theory of thin plates. For static deformation of the plate, they obtained the pull-in parameters using the shooting method and studied the small amplitude free vibration with respect to the pre-deformed bending configuration. They examined the influences of various parameters, such as the initial gap, thickness ratio, Casimir effect, and pre-stress, on the pull-in instability behavior and natural frequency. Soleymani et al. [27] studied the static pull-in instability of circular micro-plate and the effect of residual stress on pullin parameters using finite-difference method. In their model, they employed linear bending theory of thin plates and ignored the geometric nonlinearity. Nayfeh et al. [28] presented reduced-order models to study pull-in instability of beams, rectangular, and circular plates.

In literature reviews, there are limited studies on pull-in instability of annular plates, the effect of rigid core, and in-plane force. Hence, in this work, static pull-in behaviors of circular and annular plates modelled are studied according to classical plate theory using nonlinear von Kármán strain-displacement relation. This theory can account for large deflection of plate subjected to electrostatic force. By using arclength continuation method, the effects of different structural and geometrical parameters, radial force, and rigid core on pull-in instability are studied. In addition, the effects of electrostatic force, structural and geometrical parameters on natural frequencies of plate are determined.

In the following, at first, the formulation of problem consisting of equation of motion and boundary conditions is presented. Then, the solution and arclength continuation methods are described; results and discussion of the obtained equation are given. Finally, the last section concludes the paper.

\section{Statement and formulation of the problem}

The geometric structures of circular and annular plates are shown in Figure 1. In Figure 1(a), a circular plate of radius, $R$, and thickness, $h$, subjected to radial load, $P_{r}$, is depicted, and Figure $1(\mathrm{~b})$ shows a circular plate with central rigid core. The circular plate has radius, $R$, and inner radius of central core, $b$. The plate is suspended above the infinite ground plane with an initial gap, $d$. A positive potential difference, $V$, between the two conductors causes the plate to deflect.

According to the classic plate theory, the displacement field of the plate can be stated in to the following form [29]:

$$
\begin{aligned}
& u_{r}(r, \theta, z, t)=u(r, \theta, t)-z \frac{\partial w}{\partial r}, \\
& u_{\theta}(r, \theta, z, t)=v(r, \theta, t)-\frac{z}{r} \frac{\partial w}{\partial \theta}, \\
& u_{z}(r, \theta, z, t)=w(r, \theta, t) .
\end{aligned}
$$




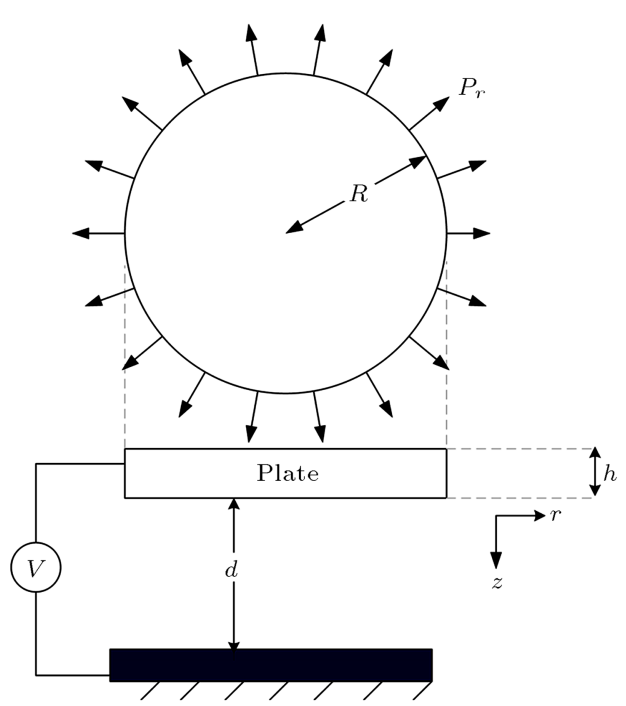

(a)

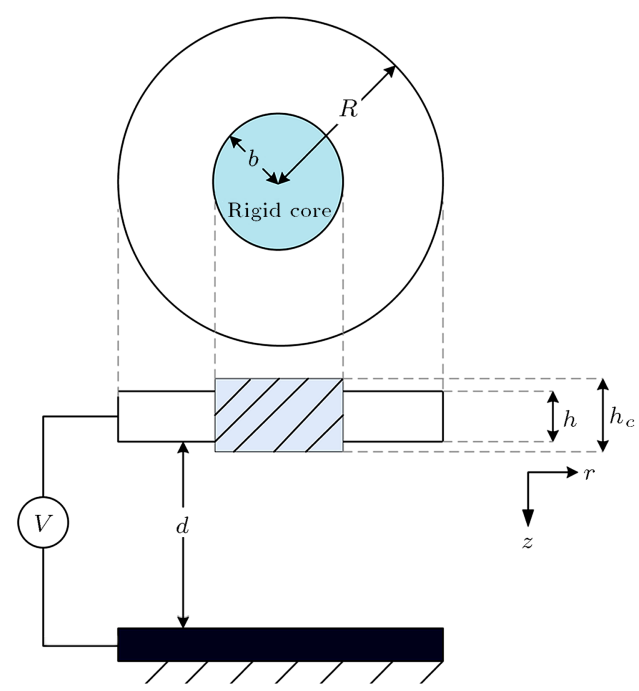

(b)

Figure 1. A schematic of electrostatically actuated plate: (a) Solid circular plate and (b) circular plate with central rigid core.

Using Eq. (1) and von Kármán strain-displacement relation and neglecting in-plane displacements, the strain components are defined as follows [29]:

$$
\begin{aligned}
& \varepsilon_{r r}=\frac{1}{2}\left(\frac{\partial w}{\partial r}\right)^{2}-z \frac{\partial^{2} w}{\partial r^{2}} \\
& \varepsilon_{\theta \theta}=\frac{1}{2 r^{2}}\left(\frac{\partial w}{\partial \theta}\right)^{2}-\frac{z}{r}\left(\frac{\partial w}{\partial r}+\frac{1}{r} \frac{\partial^{2} w}{\partial \theta^{2}}\right), \\
& 2 \varepsilon_{r \theta}=\frac{1}{r} \frac{\partial w}{\partial r} \frac{\partial w}{\partial \theta}-2 \frac{z}{r}\left(\frac{\partial^{2} w}{\partial r \partial \theta}-\frac{1}{r} \frac{\partial w}{\partial \theta}\right), \\
& \varepsilon_{z z}=\varepsilon_{\theta z}=\varepsilon_{r z}=0 .
\end{aligned}
$$

The stress and strain relations for isotropic plate are given as [29]:

$$
\begin{aligned}
\sigma_{r r} & =\frac{E}{1-\nu^{2}}\left(\epsilon_{r r}+\nu \varepsilon_{\theta \theta}\right), \\
\sigma_{\theta \theta} & =\frac{E}{1-\nu^{2}}\left(\epsilon_{\theta \theta}+\nu \epsilon_{r r}\right), \quad \sigma_{r \theta}=2 G_{\epsilon_{r \theta}},
\end{aligned}
$$

where $G=\frac{E}{2(1+\nu)}$.

The Hamilton principle is used to develop the governing equations of motion. For this purpose, the kinetic and potential energies of the system should be obtained. The kinetics and strain energies of $T$ and $\Pi$ can be expressed respectively as follows [29]:

$$
T=\frac{1}{2} \iint_{\Omega_{0}} \int_{-h / 2}^{h / 2} \rho\left(\dot{u}_{r}^{2}+\dot{u}_{\theta}^{2}+\dot{u}_{z}^{2}\right) d z r d r d \theta+T_{\text {core }}
$$

$$
\Pi=\frac{1}{2} \iint_{\Omega_{0}} \int_{-h / 2}^{h / 2}\left(\sigma_{r r} \epsilon_{r r}+\sigma_{\theta \theta} \epsilon_{\theta \theta}+\sigma_{r \theta} \gamma_{r \theta}\right) d z r d r d \theta
$$

where $\Omega_{0}$ denotes the domain of the plate. In Eq. (4), $T_{\text {core }}$ is the kinetic energy of rigid core that can be expressed as [30]:

$$
T_{\text {core }}=\frac{1}{2} M_{\text {core }}\left(\left.\frac{\partial w}{\partial t}\right|_{r=b}\right)^{2} .
$$

Due to symmetry in geometry, boundary and loading conditions of the considered plate structure in Figure 1, in the first mode of instability, the rigid core only has transverse displacement and is not necessary to include the effect of rotational kinetic energy of rigid core. The work, $W_{p}$, of radial force, $P_{r}$, can be expressed as in the following form [31]:

$$
W_{p}=\iint_{\Omega_{0}} P_{r} \varepsilon_{r r} r d r d \theta
$$

The electrostatic force per unit area between two conductive plates is given by [32]:

$$
f_{e s}=\frac{1}{2} \frac{\epsilon V^{2}}{(d-w)^{2}},
$$

where $V$ is the applied DC voltage. The work of electrostatic force, $W_{f e}$, can be expressed as [33]:

$$
W_{f e}=\iint_{\Omega_{0}} f_{e s} w r d r d \theta
$$

Then, the total work is given as in the following form:

$$
W=W_{p}+W_{f e} .
$$


It is assumed that the plate has structural damping. The work of distributed structural damping, $W_{c}$, can be expressed as follows [33]:

$$
W_{c}=\iint_{\Omega_{0}} c \frac{\partial w}{\partial t} w r d r d \theta
$$

By substituting Eqs. (4)-(11) into extended Hamilton principle and setting the coefficients of $\delta w$ in the area integrand equal to zero, equation of motion will be obtained as follows (see the Appendix):

$$
\begin{gathered}
-\frac{\partial}{\partial r}\left(r N_{r r} \frac{\partial w}{\partial r}\right)-\frac{1}{r} \frac{\partial}{\partial \theta}\left(N_{\theta \theta} \frac{\partial w}{\partial \theta}\right)-\frac{\partial}{\partial r}\left(N_{r \theta} \frac{\partial w}{\partial \theta}\right) \\
-\frac{\partial}{\partial \theta}\left(N_{r \theta} \frac{\partial w}{\partial r}\right)-\frac{\partial^{2}\left(r M_{r r}\right)}{\partial r^{2}}+\frac{\partial M_{\theta \theta}}{\partial r} \\
-\frac{1}{r} \frac{\partial^{2} M_{\theta \theta}}{\partial \theta^{2}}-2 \frac{\partial^{2} M_{r \theta}}{\partial r \partial \theta}-\frac{2}{r} \frac{\partial M_{r \theta}}{\partial \theta} \\
-P_{r} r\left(\frac{\partial^{2} w}{\partial r^{2}}+\frac{1}{r} \frac{\partial w}{\partial r}\right)+r I_{0} \ddot{w}+r c \dot{w} \\
I_{2} \frac{\partial^{2}}{\partial t^{2}}\left[\frac{\partial}{\partial r}\left(r \frac{\partial w}{\partial r}\right)+\frac{1}{r} \frac{\partial^{2} w}{\partial \theta^{2}}\right]-\frac{r \epsilon V^{2}}{2(d-w)^{2}}=0
\end{gathered}
$$

where $I_{0}=\rho h$ is the mass per unit area, and $I_{2}=\frac{\rho h^{3}}{12}$ is the rotary inertia. Boundary conditions are:

$$
\begin{gathered}
{\left[r N_{r r} \frac{\partial w}{\partial r}+N_{r \theta} \frac{\partial w}{\partial \theta}+\frac{\partial\left(r M_{r r}\right)}{\partial r}-M_{\theta \theta}\right.} \\
\left.+r I_{2} \frac{\partial^{3} w}{\partial r \partial t^{2}}+\frac{\partial M_{r \theta}}{\partial \theta}\right]_{r_{2}} \delta w\left(r_{2}, t\right)=0 \\
{\left[r N_{r r} \frac{\partial w}{\partial r}+N_{r \theta} \frac{\partial w}{\partial \theta}+\frac{\partial\left(r M_{r r}\right)}{\partial r}-M_{\theta \theta}\right.} \\
\quad+r I_{2} \frac{\partial^{3} w}{\partial r \partial t^{2}}+\frac{\partial M_{r \theta}}{\partial \theta} \\
\left.-M_{\operatorname{core}} \frac{\partial^{2} w(b)}{\partial t^{2}}\right]_{r_{1}}^{\delta w}\left(r_{1}, t\right)=0 \\
{\left[\frac{N_{\theta \theta}}{2 r} \frac{\partial w}{\partial \theta}+N_{r \theta} \frac{\partial w}{\partial r}+\frac{\partial}{\partial \theta}\left(\frac{M_{\theta \theta}}{r}\right)+\frac{2}{r} M_{r \theta}\right.} \\
\left.\quad+\frac{I_{2}}{r} \frac{\partial^{3} w}{\partial t^{2} \partial \theta}+\frac{\partial M_{r \theta}}{\partial r}\right]\left.\partial w\right|_{\theta_{1}} ^{\theta_{2}}=0 \\
-\left.r M_{r r} \delta\left(\frac{\partial w}{\partial r}\right)\right|_{r_{1}} ^{r_{2}}=0 \\
=
\end{gathered}
$$

$$
\begin{aligned}
& -\left.\frac{M_{\theta \theta}}{r} \delta\left(\frac{\delta w}{\delta \theta}\right)\right|_{\theta_{1}} ^{\theta_{2}}=0, \\
& -\left.\left.2 M_{r \theta} \delta\left(\frac{\delta w}{\delta r}\right)\right|_{\theta_{1}} ^{\theta_{2}}\right|_{r_{1}} ^{r_{2}}+\left.\left.\int_{r_{1}}^{r_{2}} \frac{\partial M_{r \theta}}{\partial r}\right|_{\theta_{1}} ^{\theta_{2}} \delta w\right|_{\theta_{1}} ^{\theta_{2}} d r \\
& +\left.\left.\int_{\theta_{1}}^{\theta_{2}} \frac{\partial M_{r \theta}}{\partial \theta}\right|_{r_{1}} ^{r_{2}} \delta w\right|_{r_{1}} ^{r_{2}} d \theta=0,
\end{aligned}
$$

where $r_{1}$ and $r_{2}$ are inner and outer radii, and $\theta_{1}$ and $\theta_{2}$ are the angles of circular sectors. For circular (annular) plate, $r_{1}=0(b), r_{2}=R, \theta_{1}=0$, and $\theta_{2}=2 \pi$. Further, $N_{r r}, N_{\theta \theta}$, and $N_{r \theta}$ are in-plane forces and $M_{r r}, M_{\theta \theta}$, and $M_{r \theta}$ are out-of-plane moments known as the stress resultants (see Figure 2). These terms are defined as follows [29]:

$$
\begin{aligned}
N_{r r} & =\int_{-h / 2}^{h / 2} \sigma_{r r} d z, \quad N_{\theta \theta}=\int_{-h / 2}^{h / 2} \sigma_{\theta \theta} d z, \\
N_{r \theta} & =\int_{-h / 2}^{h / 2} \sigma_{r \theta} d z, \\
M_{r r}= & \int_{-h / 2}^{h / 2} \sigma_{r r} z d z, \quad M_{\theta \theta}=\int_{-h / 2}^{h / 2} \sigma_{\theta \theta} z d z, \\
M_{r \theta}= & \int_{-h / 2}^{h / 2} \sigma_{r \theta} z d z .
\end{aligned}
$$

By substituting Eqs. (2) and (3) into Eq. (14), these

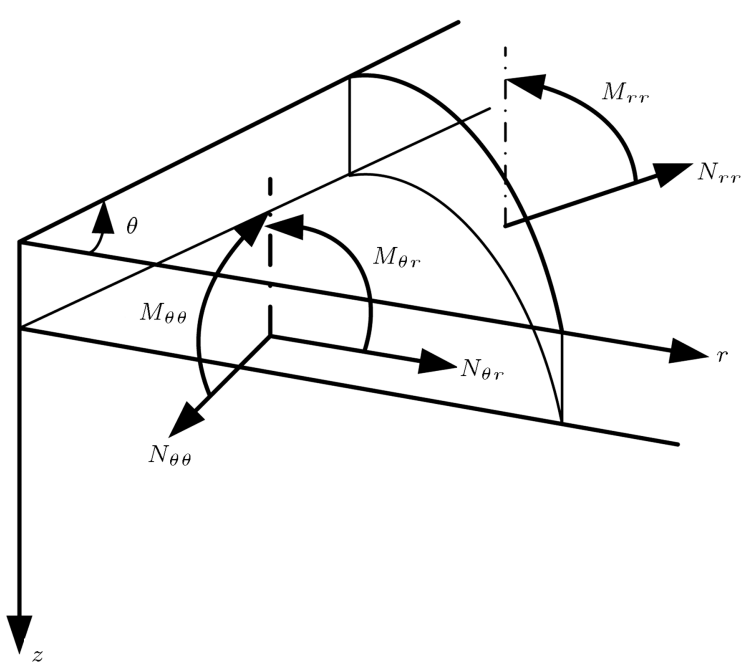

Figure 2. In-plane and out-of-plane forces and moments on a plate element. 
forces and moments will be obtained as follows:

$$
\begin{aligned}
N_{r r}= & \frac{E h}{1-\nu^{2}}\left[\frac{1}{2}\left(\frac{\partial w}{\partial r}\right)^{2}+\nu \frac{1}{2 r^{2}}\left(\frac{\partial w}{\partial \theta}\right)^{2}\right] \\
N_{\theta \theta}= & \frac{E h}{1-\nu^{2}}\left[\frac{1}{2 r^{2}}\left(\frac{\partial w}{\partial \theta}\right)^{2}+\nu \frac{1}{2}\left(\frac{\partial w}{\partial r}\right)^{2}\right] \\
N_{r \theta}= & \operatorname{Gh} \frac{1}{r} \frac{\partial w}{\partial r} \frac{\partial w}{\partial \theta}, \\
M_{r r}= & -\frac{E h^{3}}{12\left(1-\nu^{2}\right)}\left[\frac{\partial^{2} w}{\partial r^{2}}+\frac{\nu}{r}\left(\frac{\partial w}{\partial r}+\frac{1}{r} \frac{\partial^{2} w}{\partial \theta^{2}}\right)\right] \\
M_{\theta \theta}= & -\frac{E h^{3}}{12\left(1-\nu^{2}\right)}\left[\frac{1}{r}\left(\frac{\partial w}{\partial r}+\frac{1}{r} \frac{\partial^{2} w}{\partial \theta^{2}}\right)\right. \\
& \left.+\nu \frac{\partial^{2} w}{\partial r^{2}}\right], \\
M_{r \theta}= & -\mathrm{Gh}^{3} \frac{2}{12 r}\left(\frac{\partial^{2} w}{\partial r \partial \theta}-\frac{1}{r} \frac{\partial w}{\partial \theta}\right) .
\end{aligned}
$$

Galerkin's method is used for discretizing partial differential equation and discretizing them to a system of coupled nonlinear Ordinary Differential Equations (ODEs). In Galerkin's method, displacement is written in terms of time-dependent generalized coordinate and spatial (trial) function. The trial function must satisfy all geometric and natural boundary conditions of the problem. For the considered problem, natural boundary conditions are nonlinear; hence, selecting spatial function to satisfy these boundary conditions is very hard. Hence, a better way to challenge nonlinear natural boundary conditions is to include the related work into the equation of motion. With this strategy in inducing the work done by natural boundary conditions into equation of motion, it is only necessary for the trial function to satisfy geometric boundary conditions.

According to Eq. (12), the equation of motion has a fractional term of $\frac{r \epsilon V^{2}}{2(d-w)^{2}}$. Hence, to remedy the complicated form of equation of motion, the whole equation is multiplied by $(d-w)^{2} \delta w d r$. Then, the obtained equation is integrated into the whole region of plate, and as previously described, for satisfying natural boundary conditions, the work done by boundary terms is added to the weighted residual form of equation of motion. The following motion equation, in terms of displacements, will be calculated. This equation can be calculated by considering an axisymmetric solution:

$$
\begin{aligned}
& \int_{r_{1}}^{r_{2}}\left\{-\frac{E h}{2\left(1-\nu^{2}\right)}\left[\left(\frac{\partial w}{\partial r}\right)^{2} \frac{\partial w}{\partial r}+3 r\left(\frac{\partial w}{\partial r}\right)^{2} \frac{\partial^{2} w}{\partial r^{2}}\right]\right. \\
& +\frac{E h^{3}}{12\left(1-\nu^{2}\right)} \frac{\partial}{\partial r}\left(\frac{\partial^{2} w}{\partial r^{2}}+r \frac{\partial^{3} w}{\partial r^{3}}+\nu \frac{\partial^{2} w}{\partial r^{2}}\right) \\
& -\frac{E h^{3}}{12\left(1-\nu^{2}\right)}\left[-\frac{1}{r^{2}} \frac{\partial w}{\partial r}+\frac{1}{r} \frac{\partial^{2} w}{\partial r^{2}}+\nu \frac{\partial^{3} w}{\partial r^{3}}\right] \\
& -P_{r} r\left(\frac{\partial^{2} w}{\partial r^{2}}+\frac{1}{r} \frac{\partial w}{\partial r}\right)+r I_{0} \ddot{w}+r c \dot{w} \\
& \left.-I_{2} \frac{\partial^{2}}{\partial t^{2}}\left[\frac{\partial}{\partial r}\left(r \frac{\partial w}{\partial r}\right)\right]\right\}(d-w)^{2} \delta w d r \\
& -\int_{r_{1}}^{r_{2}} \frac{r \epsilon V^{2}}{2} \delta w d r+d^{2}\left[\left.\frac{1}{2} \frac{E h}{1-\nu^{2}} r\left(\frac{\partial w}{\partial r}\right)^{2} \frac{\partial w}{\partial r} \delta w\right|_{r_{1}} ^{r_{2}}\right. \\
& +\left.\frac{E h^{3}}{12\left(1-\nu^{2}\right)} r\left[\frac{\partial^{2} w}{\partial r^{2}}+\frac{\nu}{r} \frac{\partial w}{\partial r}\right] \delta\left(\frac{\partial w}{\partial r}\right)\right|_{r_{1}} ^{r_{2}} \\
& +\left.\frac{E h^{3}}{12\left(1-\nu^{2}\right)}\left[\frac{1}{r} \frac{\partial w}{\partial r}+\nu \frac{\partial^{2} w}{\partial r^{2}}\right] \delta w\right|_{r_{1}} ^{r_{2}} \\
& \left.-\left.\frac{\partial}{\partial r}\left(r \frac{E h^{3}}{12\left(1-\nu^{2}\right)}\left[\frac{\partial^{2} w}{\partial r^{2}}+\frac{\nu}{r} \frac{\partial w}{\partial r}\right]\right) \delta w\right|_{r_{1}} ^{r_{2}}\right]=0
\end{aligned}
$$

The following dimensionless variables are introduced:

$$
\begin{aligned}
& \bar{r}=\frac{r}{R}, \quad \bar{w}=\frac{w}{d}, \quad \alpha_{1}=\frac{6 d^{2}}{h^{2}}, \quad \alpha_{2}=\frac{\epsilon R^{4}}{2 D d^{3}}, \\
& \bar{V}=\alpha_{2} V^{2}, \quad \bar{t}=\frac{1}{R^{2}}\left(\frac{D}{\rho h}\right)^{\frac{1}{2}} t, \\
& I_{d}=\frac{I_{2}}{I_{0} R^{2}}, \quad \bar{P}_{r}=\frac{R^{2}}{D} P_{r},
\end{aligned}
$$

where $D=\frac{E h^{3}}{12\left(1-\nu^{2}\right)}$. The dimensionless nonlinear governing equation of motion can be obtained in the following form:

$$
\begin{aligned}
& \int_{r_{1}}^{r_{2}}\left\{-\alpha_{1}\left(\left(\frac{\partial \bar{w}}{\partial \bar{r}}\right)^{3}+3 \bar{r}\left(\frac{\partial \bar{w}}{\partial \bar{r}}\right)^{2} \frac{\partial^{2} \bar{w}}{\partial \bar{r}^{2}}\right)\right. \\
& +\left(\bar{r} \frac{\partial^{4} \bar{w}}{\partial \bar{r}^{4}}+2 \frac{\partial^{3} \bar{w}}{\partial \bar{r}^{3}}-\frac{1}{\bar{r}} \frac{\partial^{2} \bar{w}}{\partial \bar{r}^{2}}+\frac{1}{\bar{r}^{2}} \frac{\partial \bar{w}}{\partial \bar{r}}\right) \\
& -\bar{r} \bar{P}_{r}\left(\frac{\partial^{2} \bar{w}}{\partial \bar{r}^{2}}+\frac{1}{\bar{r}} \frac{\partial \bar{w}}{\partial \bar{r}}\right)+\bar{r} \frac{\partial^{2} \bar{w}}{\partial \bar{t}^{2}}+\bar{c} \bar{r} \frac{\partial \bar{w}}{\partial \bar{t}}
\end{aligned}
$$




$$
\begin{aligned}
& \left.-I_{d} \frac{\partial^{2}}{\partial \bar{t}^{2}}\left(\frac{\partial \bar{w}}{\partial \bar{r}}+\bar{r} \frac{\partial^{2} \bar{w}}{\partial \bar{r}^{2}}\right)\right\}(1-\bar{w})^{2} \delta \bar{w} d \bar{r} \\
& -\int_{r_{1}}^{r_{2}} \bar{r} \bar{V} \delta \bar{w} d \bar{r}+\left.\alpha_{1} \bar{r}\left(\frac{\partial \bar{w}}{\partial \bar{r}}\right)^{3} \delta \bar{w}\right|_{r_{1}} ^{r_{2}}+\bar{r}\left[\frac{\partial^{2} \bar{w}}{\partial \bar{r}^{2}}\right. \\
& \left.+\frac{\nu}{\bar{r}} \frac{\partial \bar{w}}{\partial \bar{r}}\right]\left.\frac{\partial \delta \bar{w}}{\partial \bar{r}}\right|_{r_{1}} ^{r_{2}}+\left.\left[\frac{1}{\bar{r}} \frac{\partial \bar{w}}{\partial \bar{r}}+\nu \frac{\partial^{2} \bar{w}}{\partial \bar{r}^{2}}\right] \delta \bar{w}\right|_{r_{1}} ^{r_{2}} \\
& -\left.\frac{\partial}{\partial \bar{r}}\left(\bar{r}\left[\frac{\partial^{2} \bar{w}}{\partial \bar{r}^{2}}+\frac{\nu}{r} \frac{\partial \bar{w}}{\partial \bar{r}}\right]\right) \delta \bar{w}\right|_{r_{1}} ^{r_{2}}=0 .
\end{aligned}
$$

As mentioned previously, in order to discretize partial differential motion equation to a system of coupled ODEs, Galerkin's method is used. In Galerkin's procedure, $\bar{w}(\bar{r}, \bar{t})$ can be written as follows:

$$
\bar{w}(\bar{r}, \bar{t})=\sum_{j=1}^{N} q_{j}(\bar{t}) \phi_{j}(\bar{r})
$$

where $q_{j}(\bar{t})$ is the time-dependent generalized coordinates, $\phi_{j}(\bar{r})$ is the $j$ th axisymmetric linear un-damped mode shape of the flat circular and annular plate, and $N$ represents the number of modes retained in the solution. $\phi_{j}(\bar{r})$ has the following general form [33].

$$
\begin{aligned}
\phi_{j}(\bar{r})= & C_{1} J_{0}\left(\lambda_{j} \bar{r}\right)+C_{2} Y_{0}\left(\lambda_{j} \bar{r}\right) \\
& +C_{3} I_{0}\left(\lambda_{j} \bar{r}\right)+C_{4} K_{0}\left(\lambda_{j} \bar{r}\right) .
\end{aligned}
$$

$J_{0}$ and $Y_{0}$ are Bessel functions of the first and seconds kinds, respectively, and $I_{0}$ and $K_{0}$ are modified Bessel functions of the first and second kinds, respectively. $C_{1}, \ldots, C_{4}$ and $\lambda$ are determined from boundary conditions.

The following nonlinear system of ODEs can be derived by substituting Eq. (19) into Eq. (18):

$$
\begin{aligned}
& \sum_{j=1}^{N} q_{j} \int_{r_{1}}^{r_{2}} \phi_{i}\left[\bar{r} \phi_{j}^{i v}+2 \phi_{j}^{\prime \prime \prime}-\frac{1}{\bar{r}} \phi_{j}^{\prime \prime}+\frac{1}{\bar{r}^{2}} \phi_{j}^{\prime}-\bar{P}_{r}\left(\bar{r} \phi_{j}^{\prime \prime}+\phi_{j}^{\prime}\right)\right] d \bar{r} \\
& \quad+\sum_{j=1}^{N} \ddot{q}_{j} \int_{r_{1}}^{r_{2}} \phi_{i}\left[\bar{r} \phi_{j}-I_{d}\left(\phi_{j}^{\prime}+\bar{r} \phi_{j}^{\prime \prime}\right)\right] d \bar{r} \\
& \quad+\bar{c} \sum_{j=1}^{\infty} \dot{q}_{j} \int_{r_{1}}^{r_{2}} \bar{r} \phi_{i} \phi_{j} d \bar{r}-2 \sum_{j=1}^{N} \sum_{k=1}^{N} q_{j} q_{k} \int_{r_{1}}^{r_{2}} \phi_{i}\left[\bar{r} \phi_{j}^{i v} \phi_{k}\right. \\
& \quad+2 \phi_{j}^{\prime \prime \prime} \phi_{k}-\frac{1}{\bar{r}} \phi_{j}^{\prime \prime} \phi_{k} \\
& \left.\quad+\frac{1}{\bar{r}^{2}} \phi_{j}^{\prime} \phi_{k}-\bar{P}_{r}\left(\bar{r} \phi_{j}^{\prime \prime} \phi_{k}+\phi_{j}^{\prime} \phi_{k}\right)\right] d \bar{r}
\end{aligned}
$$

$$
\begin{aligned}
& -2 \sum_{j=1}^{N} \sum_{k=1}^{N} q_{j} \ddot{q}_{k} \int_{r_{1}}^{r_{2}} \phi_{i}\left[\bar{r} \phi_{j} \phi_{k}-I_{d}\left(\phi_{j} \phi_{k}^{\prime}\right.\right. \\
& \left.\left.+\bar{r} \phi_{j} \phi_{k}^{\prime \prime}\right)\right] d \bar{r}-2 \bar{c} \sum_{j=1}^{N} \sum_{k=1}^{N} q_{j} \dot{q}_{k} \int_{r_{1}}^{r_{2}} \bar{r} \phi_{i} \phi_{j} \phi_{k} d \bar{r} \\
& +\sum_{j=1}^{N} \sum_{k=1}^{N} \sum_{m=1}^{N} q_{j} q_{k} q_{m} \int_{r_{1}}^{r_{2}} \phi_{i}\left[\bar{r} \phi_{j}^{i v} \phi_{k} \phi_{m}\right. \\
& +2 \phi_{j}^{\prime \prime \prime} \phi_{k} \phi_{m}-\frac{1}{\bar{r}} \phi_{j}^{\prime \prime} \phi_{k} \phi_{m}+\frac{1}{\bar{r}^{2}} \phi_{j}^{\prime} \phi_{k} \phi_{m} \\
& -\alpha_{1} \phi_{j}^{\prime} \phi_{k}^{\prime} \phi_{m}^{\prime}-3 \alpha_{1} \bar{r} \phi_{j}^{\prime \prime} \phi_{k}^{\prime} \phi_{m}^{\prime}-\bar{P}_{r}\left(\bar{r} \phi_{j}^{\prime \prime} \phi_{k} \phi_{m}\right. \\
& \left.\left.+\phi_{j}^{\prime} \phi_{k} \phi_{m}\right)\right] d \bar{r}+\sum_{j=1}^{N} \sum_{k=1}^{N} \sum_{m=1}^{N} q_{j} q_{k} \ddot{q}_{m} \\
& \int_{r_{1}}^{r_{2}} \phi_{i}\left[\bar{r} \phi_{j} \phi_{k} \phi_{m}-I_{d}\left(\phi_{j} \phi_{k} \phi_{m}^{\prime}+\bar{r} \phi_{j} \phi_{k} \phi_{m}^{\prime \prime}\right)\right] d \bar{r} \\
& +\bar{c} \sum_{j=1}^{N} \sum_{k=1}^{N} \sum_{m=1}^{N} q_{j} q_{k} \dot{q}_{m} \int_{r_{1}}^{r_{2}} \bar{r} \phi_{i} \phi_{j} \phi_{k} \phi_{m} d \bar{r} \\
& +2 \alpha_{1} \sum_{j=1}^{N} \sum_{k=1}^{N} \sum_{m=1}^{N} \sum_{n=1}^{N} q_{j} q_{k} q_{m} q_{n} \int_{r_{1}}^{r_{2}} \phi_{i}\left[\phi_{j}^{\prime} \phi_{k}^{\prime} \phi_{m}^{\prime} \phi_{n}\right. \\
& \left.+3 \bar{r} \phi_{j}^{\prime \prime} \phi_{k}^{\prime} \phi_{m}^{\prime} \phi_{n}\right] d \bar{r} \\
& -\alpha_{1} \sum_{j=1}^{N} \sum_{k=1}^{N} \sum_{m=1}^{N} \sum_{n=1}^{N} \sum_{r=1}^{N} q_{j} q_{k} q_{m} q_{n} q_{r} \int_{r_{1}}^{r_{2}} \phi_{i}
\end{aligned}
$$$$
\left[\phi_{j}^{\prime} \phi_{k}^{\prime} \phi_{m}^{\prime} \phi_{n} \phi_{r}+3 \bar{r} \phi_{j}^{\prime \prime} \phi_{k}^{\prime} \phi_{m}^{\prime} \phi_{n} \phi_{r}\right] d \bar{r}-\int_{r_{1}}^{r_{2}} \bar{r} \bar{V} \phi_{i} d \bar{r}
$$$$
+\left.\alpha_{1} \sum_{j=1}^{N} \sum_{k=1}^{N} \sum_{m=1}^{N} q_{j} q_{k} q_{m} \bar{r} \phi_{i} \phi_{j}^{\prime} \phi_{k}^{\prime} \phi_{m}^{\prime}\right|_{r_{1}} ^{r_{2}}
$$$$
+\sum_{j=1}^{N} q_{j}\left[\bar{r} \phi_{i}^{\prime} \phi_{j}^{\prime \prime \prime}+\nu \phi_{i}^{\prime} \phi_{j}^{\prime}+\frac{1}{\bar{r}} \phi_{i} \phi_{j}^{\prime}-\bar{r} \phi_{i} \phi_{j}^{\prime \prime}\right.
$$$$
\left.-\nu \phi_{i} \phi_{j}^{\prime}-\bar{r} \phi_{i} \phi_{j}^{\prime \prime \prime}+\frac{\nu}{\bar{r}} \phi_{i} \phi_{j}^{\prime}\right]\left.\right|_{r_{1}} ^{r_{2}}=0
$$

with $i=1, \ldots, N$; in this equation, primes on $\phi$ denote derivatives with respect to $r$. 
Some necessary descriptions are required to solve the equation of motion. Due to the application of electrostatic force in MEMS, time response analysis needs stringent convergence condition, and they are stiff for time response analysis. Using arc length continuation method can obviate the need for sufficiently small-time steps. Moreover, by multiplying both sides of equation into $(d-w)^{2}$, the stiffness of the equation is reduced.

Eq. (21) is a set of nonlinear coupled equations. For solving nonlinear algebraic equations, most methods are iterative, such as continuation methods. These methods are used to compute approximate solutions for nonlinear systems with parameterized nonlinear equations. In [34], a practical guide for performing parameter studies is introduced. Numerical continuation is a tool to study how the behavior of a dynamical system changes as a function of parameters. Path following in combination with boundary value problem solvers has an important role in the development of dynamical systems [35]. Herein, the arc-length continuation method is used. In arc-length continuation method, the continuation direction is tangent to the solution branch.

To obtain deflection-voltage curves for nonlinear Eq. (21), the arc-length continuation procedure is used according to Figure $3[36,37]$.

Let the deflection-voltage curve have the following form:

$$
F(u, \lambda)=0
$$

In this method, the first step is to solve the system of nonlinear equations of Eqs. (21) or (22) for fixed values of system parameters and a very small value of applied voltage. This starting point is used as an initial guess to start the procedure of the arclength continuation method, until reaching the turning point. At the turning point, the Jacobian determinant of Eq. (22) with respect to $u$, i.e. $\partial F / \partial u$, is zero, and it is necessary to reverse the direction of change in parameter. With this procedure, the deflectionvoltage curve can be obtained with sufficient accuracy, i.e. good conformity between the results obtained by continuation method and direct solution of ordinary equations by numerical methods such as Runge-Kutta.

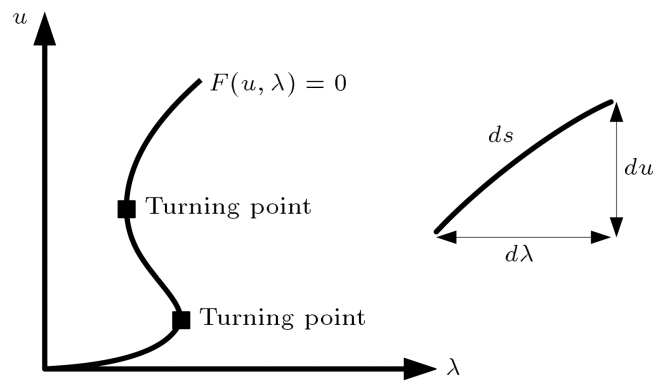

Figure 3. The deflection-voltage curve [37].

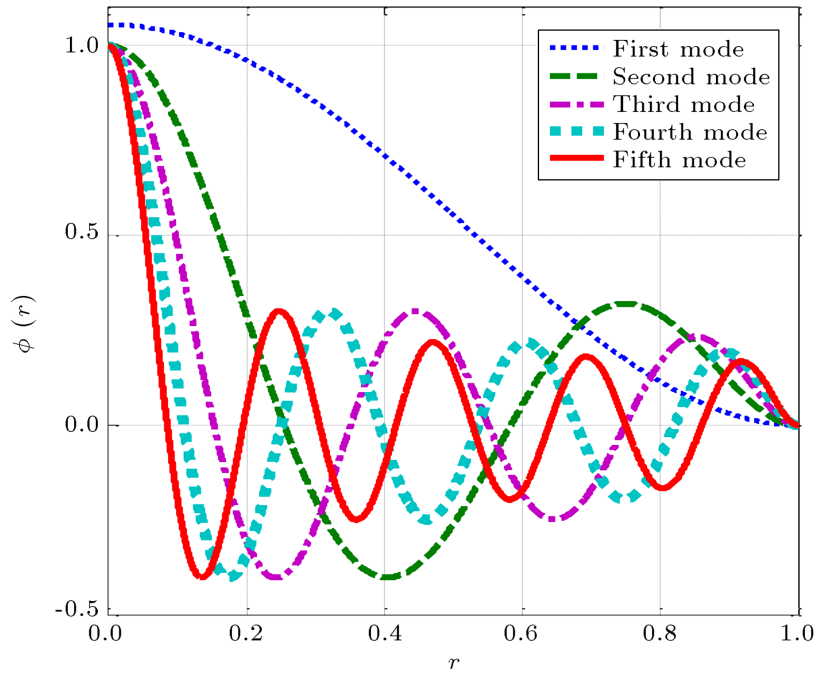

Figure 4. Mode shapes of fully clamped circular plate.

In the following part, a brief discussion of required linear mode shapes for Galerkin's method is presented. First, fully clamped circular plate is considered. The boundary conditions for clamped circular plate are:

$$
\phi(1)=0, \frac{\partial \phi(1)}{\partial \bar{r}}=0, \quad \phi(0)<\infty .
$$

The Bessel function of the second kind becomes infinite at $r=0$; therefore, constants $C_{2}$ and $C_{4}$ must be zero. Thus, Eq. (20) is simplified to:

$$
\phi(\bar{r})=C_{1} J_{0}(\lambda \bar{r})+C_{3} I_{0}(\lambda \bar{r}) .
$$

By substituting Eq. (24) into Eq. (23), the mode shapes of clamped circular plate can be obtained as follows:

$$
\phi(\bar{r})=J_{0}(\lambda \bar{r})-\frac{J_{0}(\lambda)}{J_{0}(\lambda)} I_{0}(\lambda \bar{r}) .
$$

By applying the second condition of Eqs. (23) to (25) and solving the obtained equation numerically, $\lambda$ can be obtained. In this work, the MATLAB software is used to obtain $\lambda$ and linear undamped mode. Some mode shapes of clamped circular plate are plotted in Figure 4.

The boundary conditions associated with the annular plate clamped from inside and outside edges are:

$$
\phi(1)=0, \quad \frac{\partial \phi}{\partial \bar{r}}(1)=0, \quad \phi(\beta)=0, \quad \frac{\partial \phi}{\partial \bar{r}}(\beta)=0,
$$

where $\beta=r_{i} / R$ is the dimensionless inner radius. For annular plate clamped from the inside edge and simply supported from the outside edge, the boundary conditions are:

$$
\begin{aligned}
& \phi(1)=0, \quad M_{r}=\left.D\left[\frac{\partial^{2} \phi}{\partial \bar{r}^{2}}+\nu \frac{1}{r} \frac{\partial \phi}{\partial \bar{r}}\right]\right|_{\bar{r}=1}=0, \\
& \phi(\beta)=0, \quad \frac{\partial \phi}{\partial \bar{r}}(\beta)=0 .
\end{aligned}
$$




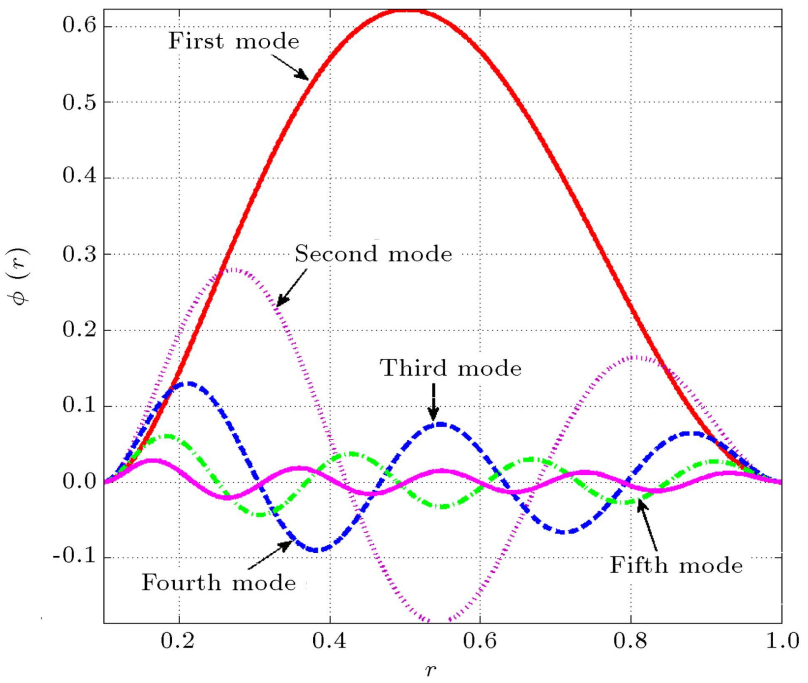

(a)

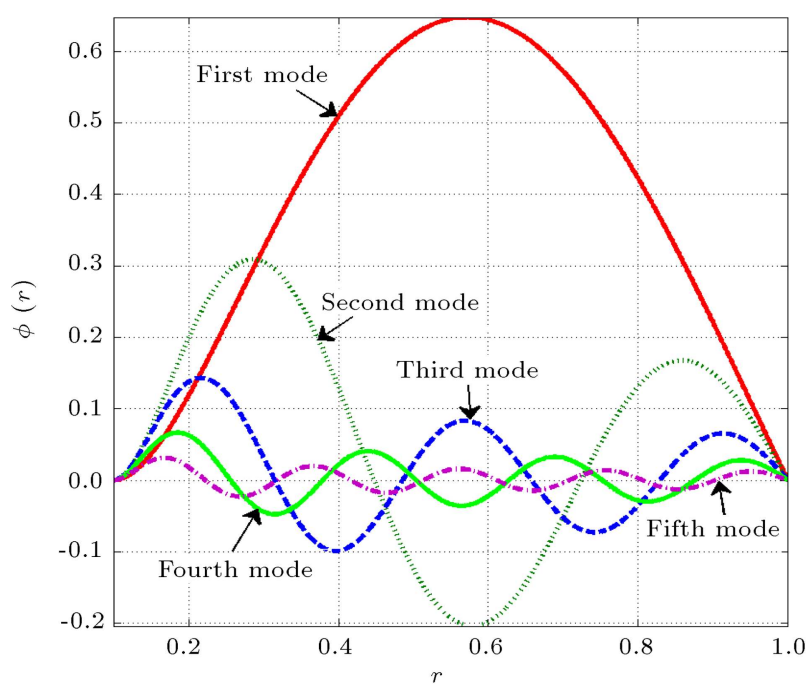

(b)

Figure 5. Mode shapes of (a) clamped-clamped and (b) clamped plates with simply supported inner and outer edges on the annular plate; $\beta=0.1, \nu=0.33$.

By substituting Eq. (20) into Eqs. (26) and (27) and numerically solving the obtained equations, the linear un-damped mode shapes of clamped-clamped and clamped-simple annular plates are determined. Figure 5 shows mode shapes of annular plates with the mentioned boundary conditions.

For a circular plate with a central rigid core, the boundary conditions are:

$$
\begin{aligned}
& \phi(1)=0, \quad \frac{\partial \phi}{\partial \bar{r}}(1)=0, \quad \frac{\partial \phi}{\partial \bar{r}}(\bar{b})=0, \\
& \left.2 \pi\left[\frac{\partial^{3} \phi}{\partial \bar{r}^{3}}+\frac{1}{\bar{r}} \frac{\partial^{2} \phi}{\partial \bar{r}^{2}}-\frac{1}{\bar{r}^{2}} \frac{\partial \phi}{\partial \bar{r}}\right]\right|_{\bar{r}=\bar{b}}=\frac{M_{c}}{R \rho h} \bar{\omega}^{2} \phi(\bar{b}),
\end{aligned}
$$

where $\bar{b}=b / R$ is the dimensionless radius of rigid core. Following the same mentioned procedure for obtaining

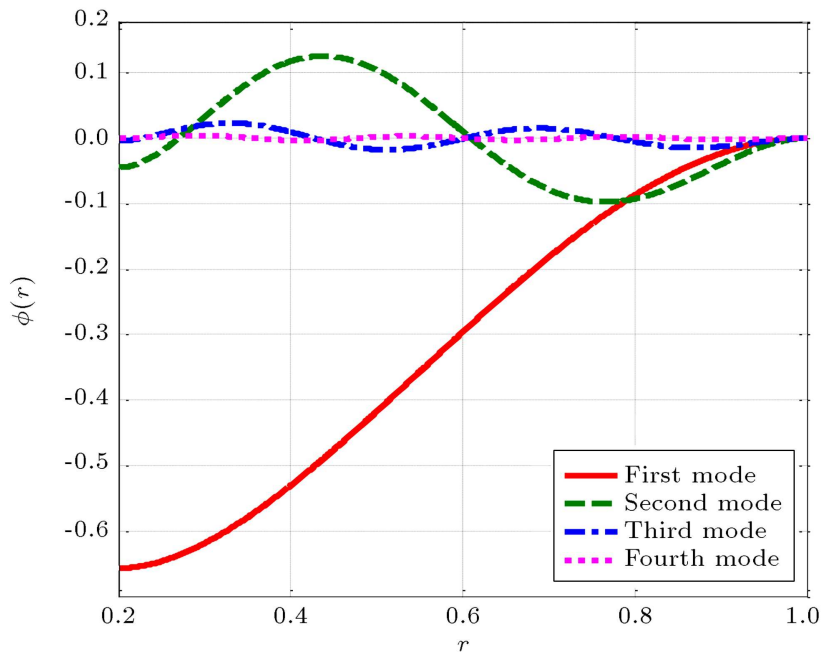

Figure 6. Mode shapes of clamped circular plate with central rigid core, $\bar{b}=0.2, \nu=0.34$.

the mode shapes of previous boundary conditions, the mode shape of this case can be calculated; however, since it requires a lengthy calculation, it is ignored here. Figure 6 shows mode shapes of clamped circular plate with central rigid core for $\bar{b}=0.2$.

\section{Results and discussion}

In this section, in order to compare the pull-in instability of plate for different boundary conditions, Eq. (21) was solved for fully clamped circular plate, clamped-clamped, and clamped-simply supported annular plates. Since the inertia terms are not important in static pull-in instability, for studding it, all timedependent terms of Eq. (21) are ignored. At first, results of clamped circular plate were compared with those presented in the literature; afterwards, static pull-in behaviors of circular and annular plates with different inner radii and boundary conditions were studied, and the effect of radial load on pull-in instability of plates was investigated. Then, the effect of applied voltage on the fundamental natural frequency of deformed plate at the state of equilibrium of the plate was studied. The effect of rigid core on the pullin stability was also accurately studied.

\subsection{Static pull-in behavior of circular and annular plates}

In this section, the static pull-in behaviors of circular and annular plates were studied. To validate the proposed model, the pull-in results of clamped circular plate were compared with the results obtained in [38], and the obtained equilibrium curve is shown in Figure 7 . The geometric and material parameters of the model were selected as Poisson's ratio $(\nu)$ of 0.25 , outer radius $(R)$ of $50 \mu \mathrm{m}$, thickness $(h)$ of $3 \mu \mathrm{m}$, initial gap of $0.894 \mu \mathrm{m}$, and permittivity of free space $(\epsilon)$ of $8.85 \times 10^{-12} \mathrm{~F} / \mathrm{m}$. 


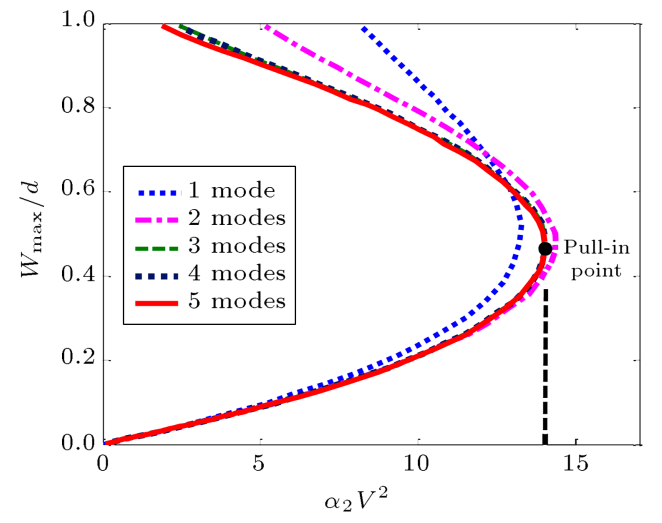

(a)

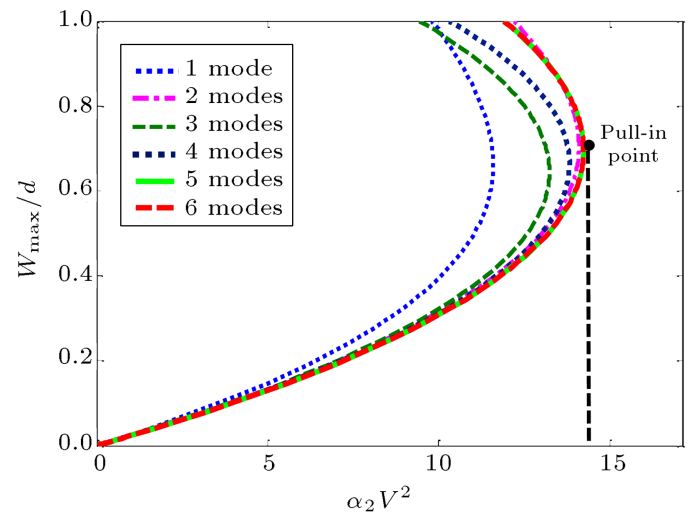

(b)

Figure 7. Equilibrium curve for clamped circular plate; comparison of (a) Ref. [38] and (b) the present work.

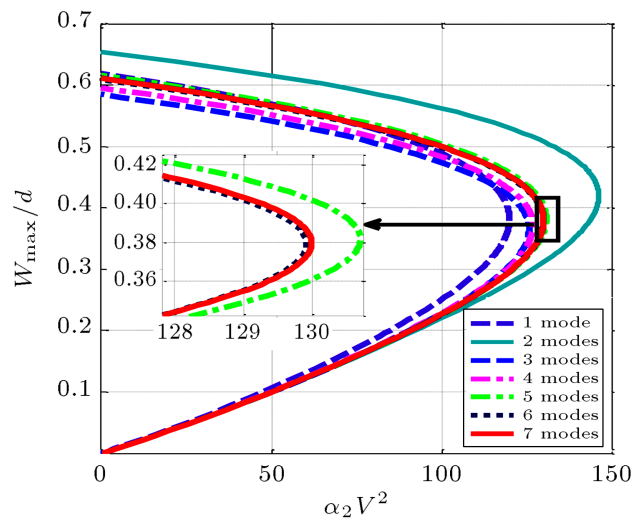

(a)

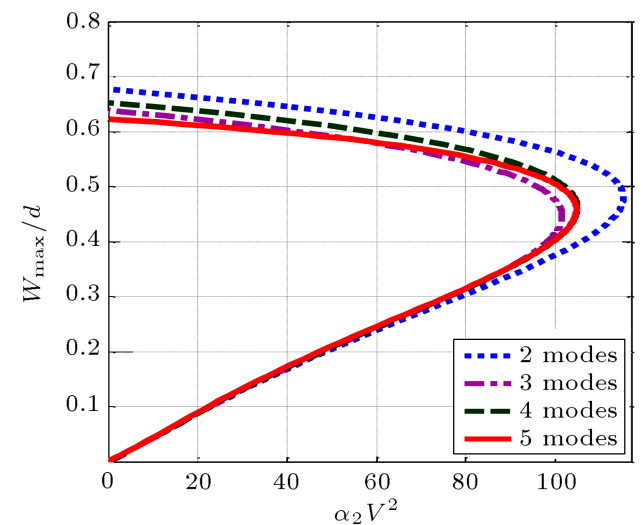

(b)

Figure 8. Equilibrium curves for (a) clamped-clamped, (b) simply supported from outside and clamped from inside annular plate with convergence of $\alpha_{1}=24, \beta=0.1$.

The saddle-node bifurcation points in Figure 7 show static pull-in instability in which stable and unstable branches of solution coincide with each other. The value of applied voltage corresponding to these points is static pull-in voltage, and the slope of all curves becomes infinite at the pull-in point. As shown in Figure 7 , the results converge as the number of modes increases; using six modes for discretizing in Galerkin's procedure can be sufficient in order to obtain accurate results. As seen in Figure 7 , using the proposed model with six modes, the estimated static pull-in voltage is 14.2, while Vogl [38] reported 14.1 as the static pull-in voltage of the plate; this shows the validity of the obtained equation and the proposed solution method.

To study the behaviors of annular plates in electrostatic field, at first, annular plate clamped from both inner and outer radii is considered, and then static behaviors of annular plates clamped from inner and simply supported from outer radii were studied. For this study, the geometric and material parameters of annular plate were selected as Poisson's ratio $(\nu)$ of
0.33 , outer radius $(R)$ of $50 \mu \mathrm{m}$, inner radius/outer radius ratio $(\beta)$ of $0.1,0.3$, and 0.5 , thickness $(h)$ of $1 \mu \mathrm{m}$, initial gap $(d)$ of $2 \mu \mathrm{m}$, and permittivity of free space $(\epsilon)$ of $8.85 \times 10^{-12} \mathrm{~F} / \mathrm{m}$.

First, it is necessary to select an appropriate number of mode shapes to describe the transverse displacement. In order to determine the number of modes to be retained in the Galerkin's procedure and to obtain accurate results, a convergence study was performed. By solving Eq. (21) for clampedclamped and clamped-simply supported annular plates with $\beta=0.1$ and $\alpha_{1}=24$, the pull-in behavior of annular plates was obtained, as shown in Figure 8, which demonstrates that using six modes is sufficient to obtain accurate results of annular plate; therefore, the following results were obtained by using six modes.

Now, after validating the equation of motion and solution method and obtaining the required number of mode shapes necessary for the Galerkin's method of solution, effects of different parameters on pullin instability were determined. Since more attention is devoted to circular plate in the literature, herein, 


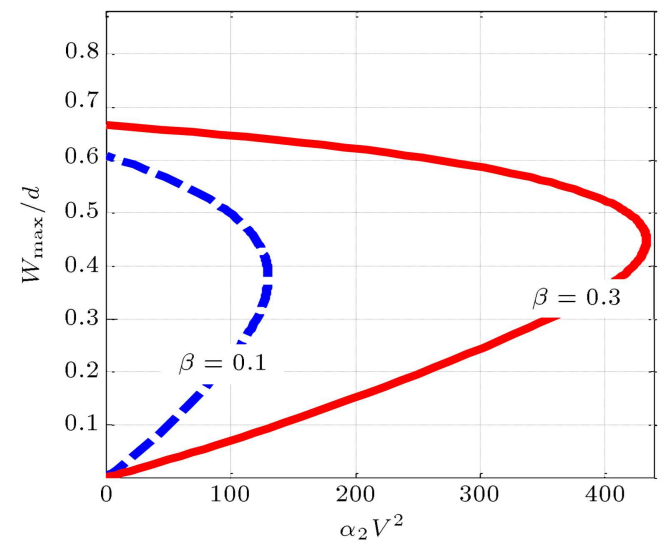

(a)

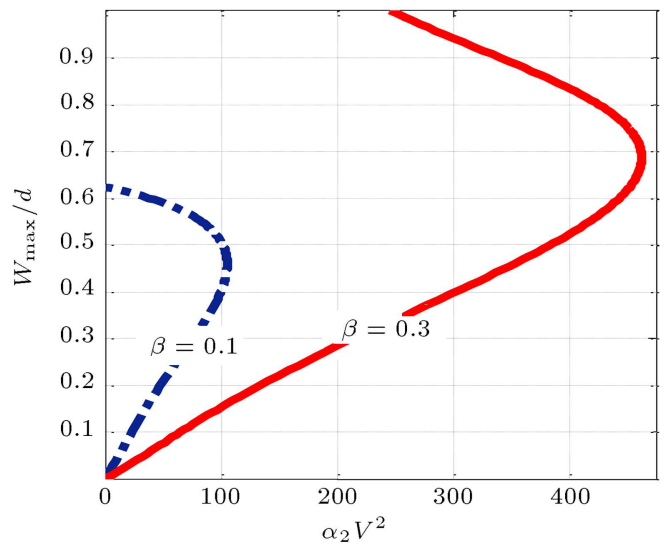

(b)

Figure 9. Equilibrium curves for annular plate with $\alpha_{1}=24$ : (a) Clamped-clamped and (b) clamped-simply supported plates; effect of inner radius.

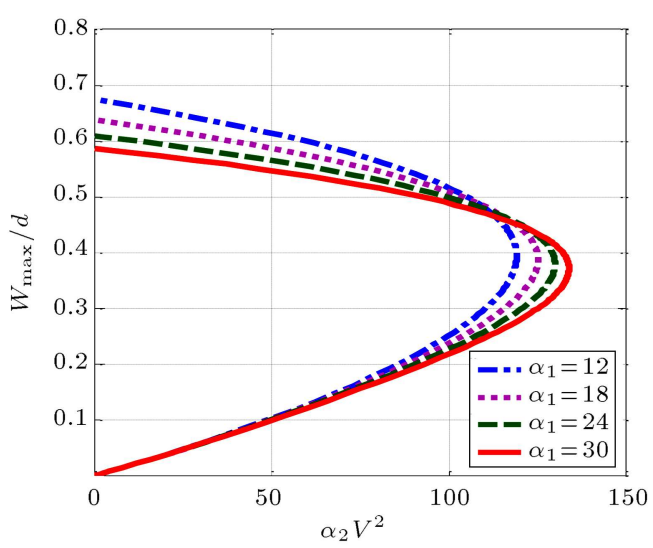

(a)

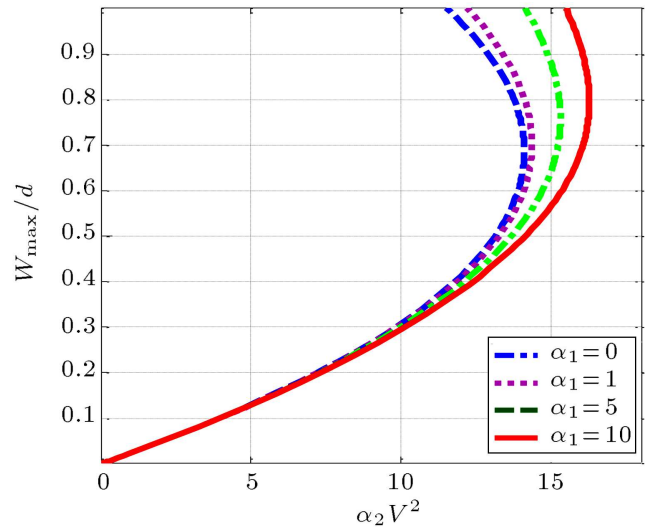

(a)

Figure 10. The effect of geometric nonlinearity on equilibrium curve for (a) clamped-clamped annular plate with $\beta=0.1$ and (b) fully clamped circular plate.

the main priority is given to annular plate. Results of clamped-clamped and clamped-simply supported annular plates with different inner radii are presented in Figure 9.

The lower branch of the obtained equilibrium curve is stable, while the upper branch is unstable. At pull-in point, a jump in the transverse deflection of plate will occur, and system will settle to its upper stable equilibrium curve (which is not shown in this figure). The distance between unstable and stable branches of equilibrium curve shows the margin of stability, since the greater the distance, the greater the margin of safety. As expected, the pull-in voltage of clamped plate is greater than that of clampedsimply supported plate. Figure 9 demonstrates that by increasing the inner radius of annular plate, pull-in voltage develops in bigger voltages and the static pullin voltage increases due to an increase in stiffness of plate.

In order to study the effect of geometric nonlinearity on the pull-in parameters, the dimensionless
Table 1. Static pull-in parameters of clamped-clamped annular plates with different geometric nonlinearity coefficients.

\begin{tabular}{cccc}
\hline $\boldsymbol{\beta}$ & $\boldsymbol{\alpha}_{\mathbf{1}}$ & $\boldsymbol{\alpha}_{\mathbf{2}} \boldsymbol{V}^{\mathbf{2}}$ & $\overline{\boldsymbol{W}}_{\max }$ \\
\hline \multirow{4}{*}{0.1} & 12 & 119.1 & 0.3942 \\
& 18 & 125.1 & 0.3876 \\
& 24 & 129.9 & 0.3794 \\
& 30 & 133.9 & 0.3711 \\
& 12 & 366 & 0.4545 \\
0.3 & 18 & 402.2 & 0.4544 \\
& 24 & 433.5 & 0.4487 \\
& 30 & 460.8 & 0.4413 \\
\hline
\end{tabular}

deflection of plate versus applied voltage was plotted for different geometric nonlinearity coefficients in Figure 10. This figure shows equilibrium curves for fully clamped circular and clamped-clamped annular plates with $\beta=0.1$ and equilibrium curves for different geometric nonlinearities. Results of other cases are presented in Table 1. 
As seen in Table 1 , by increasing $\alpha_{1}$, the static pull-in voltage increases while the deflection in pull-in voltage decreases.

\subsection{Effect of radial load on pull-in behaviors of circular and annular plates}

In this section, by applying tensile and compressive radial forces to circular and annular plates, the effects of these forces on pull-in parameters are studied. Influences of the radial force on the static pull-in voltage of circular plate with specifications, as mentioned in Figure 7, are depicted in Figure 11 for clamped circular plate. Dimensionless applied loads $\left(\bar{P}_{r}\right)$ are written on each curve. Negative numbers show compressive load, and positive ones show tensile load. As known from elasticity and vibration studies, tensile load increases stiffness, while compressive load decreases stiffness;

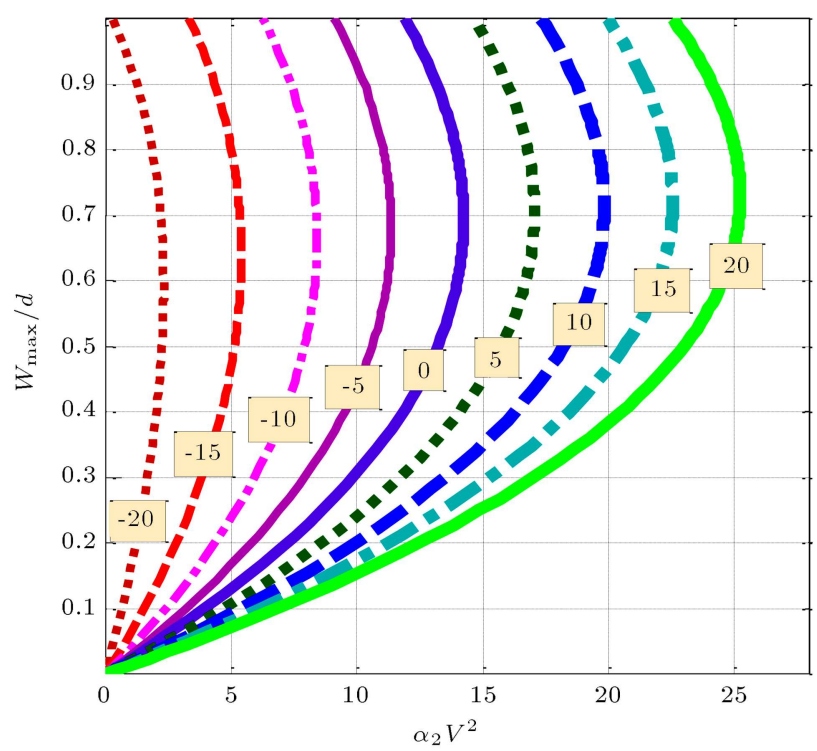

Figure 11. The effect of radial load of equilibrium curve for clamped circular plate.

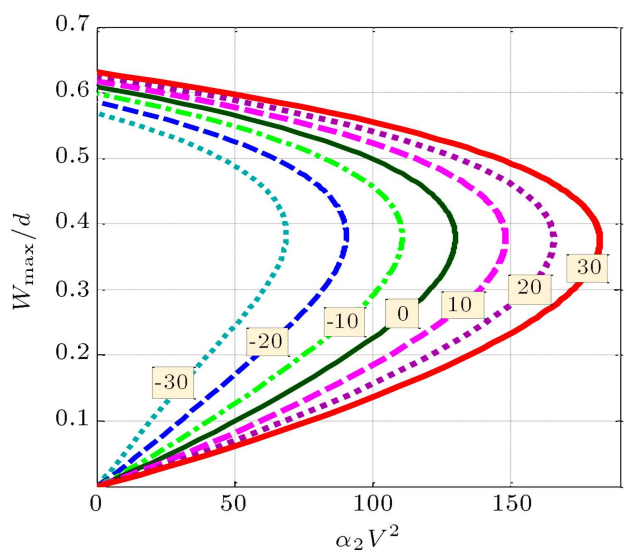

(a) accordingly, pull-in voltages increase for tensile load and decreases for compressive load.

From this figure, it can be understood that the variation of pull-in voltage with radial load is very much, while maximum deflection at the pull-in point has small variation with radial load.

In Figure 12, equilibrium curves for clampedclamped and clamped-simply supported annular plates with $\beta=0.1$ and $\alpha_{1}=24$ under different radial loads are shown. In this case, since annular plate has greater stiffness with respect to circular plate, it has greater pull-in voltages.

Results of annular plates with $\alpha_{1}=24$ and different values of $\beta$ under various radial loads are presented in Tables 2 and 3 .

As shown in Figures 11 and 12 and Tables 2 and 3 , by increasing tensile radial force, the stiffness of plate increases; thus, the static pull-in voltage of plate increases, while an increase in the value of compressive radial force has softening effect on system and decreases the stiffness, which, accordingly, decreases the static pull-in voltage of plate.

\subsection{Pull-in behavior of circular plate with the central rigid core}

In this section, the static pull-in behavior of circular plate with the central rigid core is studied. The geometric and material parameters of circular plate and rigid core are mentioned in Table 4.

Similar to the previous cases, at first, the convergence of the solution of a clamped circular plate with the central rigid core is examined in terms of the different number of mode shapes. The obtained result is shown in Figure 13. From this figure, it is clear that four mode shapes are sufficient for the convergence of solution.

According to the results shown for circular and annular plate, since the form of equilibrium curve

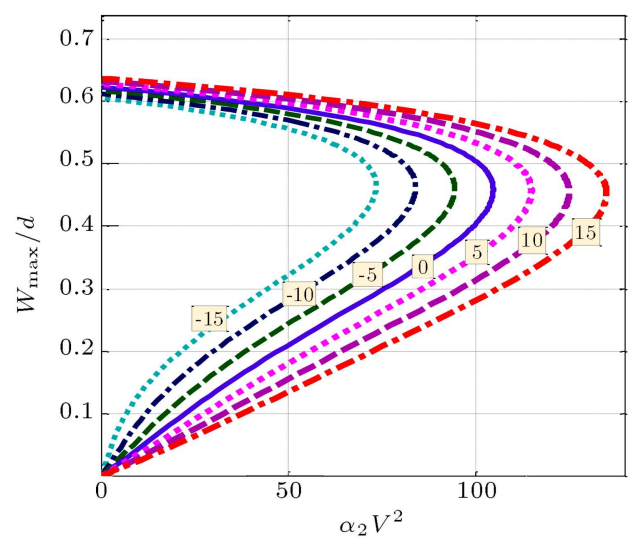

(b)

Figure 12. The effect of radial load on equilibrium curve for annular plate with $\beta=0.1$ and $\alpha_{1}=24$ : (a) Clamped-clamped, and (b) clamped-simply supported plates. 
Table 2. The effect of radial load on static pull-in voltages of clamped-simply annular plates with $\alpha_{1}=24$.

\begin{tabular}{cccccc}
\hline Radial load & $\begin{array}{c}\text { Dimensionless } \\
\text { radial load } \\
\left(\overline{\boldsymbol{P}}_{\boldsymbol{r}}\right)\end{array}$ & $\begin{array}{c}\text { Dimensionless } \\
\text { pull-in voltage } \\
\left(\boldsymbol{\alpha}_{\mathbf{2}} \boldsymbol{V}^{\mathbf{2}}\right)\end{array}$ & $\begin{array}{c}\text { Max dimensionless } \\
\text { deflection } \\
\left(\boldsymbol{W}_{\text {max }} / \boldsymbol{d}\right)\end{array}$ \\
\cline { 2 - 6 } & -30 & 68.58 & 347 & 0.3849 & 0.4608 \\
Compressive & -20 & 90.31 & 376 & 0.3827 & 0.4569 \\
& -10 & 110.7 & 404.8 & 0.381 & 0.4535 \\
& 0 & 129.9 & 433.5 & 0.379 & 0.4487 \\
Tensile & 10 & 148.2 & 462 & 0.3772 & 0.4447 \\
& 20 & 165.6 & 490.4 & 0.3754 & 0.4407 \\
& 30 & 182.4 & 518.7 & 0.3742 & 0.4367 \\
\hline
\end{tabular}

Table 3. The effect of radial load on static pull-in voltages of clamped-simply supported annular plates with $\alpha_{1}=24$.

\begin{tabular}{cccccc}
\hline Radial load & $\begin{array}{c}\text { Dimensionless } \\
\text { radial load } \\
\left(\overline{\boldsymbol{P}}_{\boldsymbol{r}}\right)\end{array}$ & $\begin{array}{c}\text { Dimensionless } \\
\text { pull-in voltage } \\
\left(\boldsymbol{\alpha}_{\mathbf{2}} \boldsymbol{V}^{\mathbf{2}}\right)\end{array}$ & $\begin{array}{c}\text { Max dimensionless } \\
\text { deflection } \\
\left(\boldsymbol{W}_{\text {max }} / \boldsymbol{d}\right)\end{array}$ \\
\cline { 2 - 6 } Compressive & -15 & 73.45 & 408.1 & 0.4646 & 0.6889 \\
& -10 & 83.99 & 426.1 & 0.4625 & 0.6881 \\
& -5 & 94.41 & 444 & 0.4604 & 0.6873 \\
& 0 & 104.7 & 462 & 0.4584 & 0.6865 \\
Tensile & 5 & 114.9 & 480 & 0.4564 & 0.6857 \\
& 10 & 125 & 498 & 0.4544 & 0.6849 \\
& 15 & 135 & 516.1 & 0.4524 & 0.6841 \\
& 20 & 144.9 & 534.1 & 0.4505 & 0.6832 \\
\hline
\end{tabular}

Table 4. The geometric and material parameters of circular plate with the central rigid core.

\begin{tabular}{lc}
\hline Parameters & Values \\
\hline Radius of plate $(R)$ & $50 \mu \mathrm{m}$ \\
Radius of core $(b)$ & $20 \mu \mathrm{m}$ \\
Thickness of plate $(h)$ & $1 \mu \mathrm{m}$ \\
Thickness of core $\left(h_{c}\right)$ & $2 \mu \mathrm{m}$ \\
Initial gap $(d)$ & $2 \mu \mathrm{m}$ \\
Density of plate $(\rho)$ & $2700 \mathrm{~kg} / \mathrm{m}^{3}$ \\
Density of core $\left(\rho_{c}\right)$ & $7800 \mathrm{~kg} / \mathrm{m}^{3}$ \\
Poisson's ratio $(\nu)$ & 0.34 \\
\hline
\end{tabular}

is similar in different cases, herein, only one case is examined. Figure 14 represents the results determined for clamped solid circular and clamped circular plates with central rigid mass.

As shown in Figure 14, the attachment of rigid core to different radius ratios has different effects on pull-in voltage. Generally, due to the attachment of rigid core, there is a reduction in the stiffness of plate. Hence, due to the reduction in the stiffness of structure, pull-in voltage reduces, which is the reason for the reduction in pull-in voltage in $b / R=0.1$ and

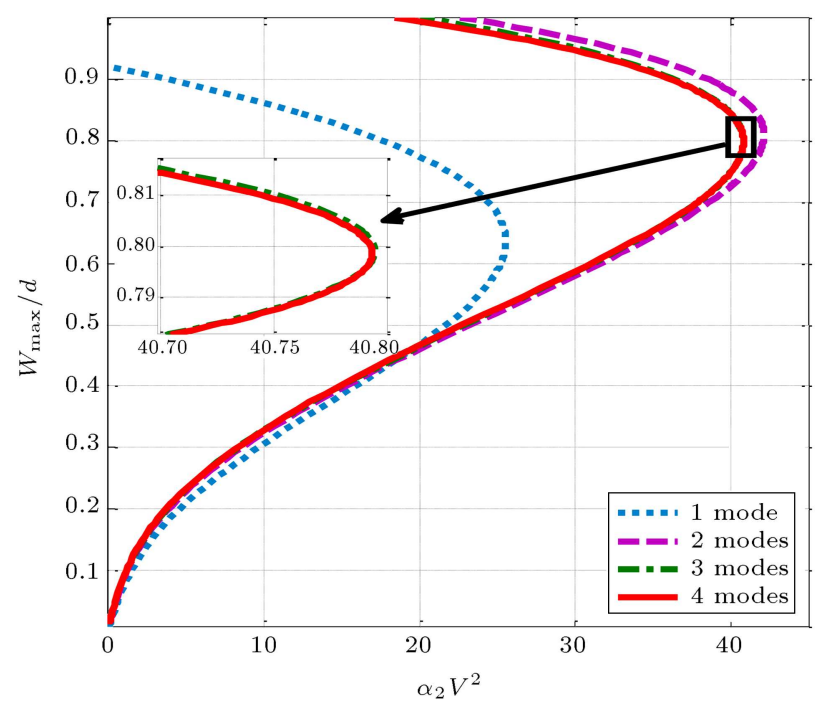

Figure 13. Equilibrium curves for clamped circular plate with the central rigid core with the convergence of $\alpha_{1}=24, b / R=0.2$.

$b / R=0.15$. However, this trend is not monotone with an increase in the radius of rigid core. In circular plate without rigid core, the deflection at the center of rigid core increases with applied voltage with no 


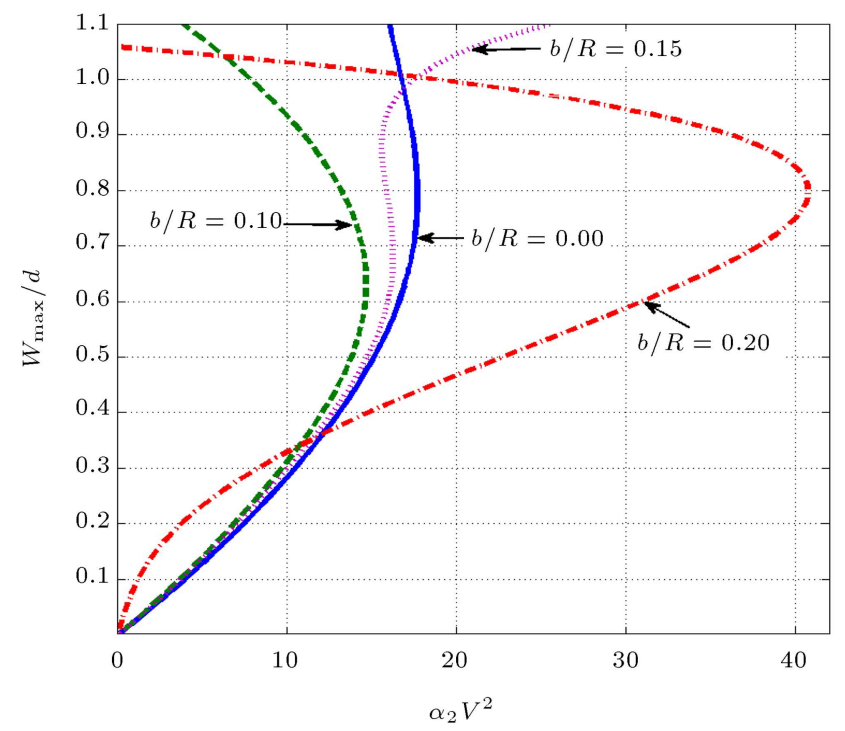

Figure 14. Comparison of equilibrium curves for clamped circular and circular plates with the central rigid core; $\alpha_{1}=24$.

limitation on it, since the whole structure has the same deformation. However, when the rigid core is attached to plate, there is limitation on the deflection at the center point, since all points in the domain of rigid core have the same displacement. The limitation on the displacement of the rigid core will be more stringent with an increase in the deflection of plate. Hence, although the plate with a rigid core has lower stiffness at its first initial shape, its rigidity will continuously increase with the deflection at center. This is the reason for an increase in pull-in voltage for $b / R=0.2$.

\subsection{Natural frequencies of plate in the electrostatic field}

When the plate is deflected, the natural frequencies change correspondingly. With the increase in applied voltage, the stiffness of plate reduces continuously, until the stiffness in the first mode of pull-in instability becomes zero in pull-in voltage, and the natural frequency becomes zero, accordingly. Hence, in the following, reduction in the natural frequency of this deformed plate at every deflection shape of plate due to electrostatic force is determined. To study the linear natural frequency of vibration, it is necessary to linearize the plate equations of motion around the deflected position.

Figures 15-17 show the variation of fundamental natural frequency of the deflected plate, normalized with respect to the natural frequency at the unreformed state along with the variation in the electrostatic load for different ratios of $\alpha_{1}$ (i.e. the geometric nonlinearity term); the radius ratio of $\beta$ is considered, too.

As shown in Figures 15-17, for low values of $\alpha_{1}$, when electrostatic load increases, the fundamental natural frequency decreases and approaches zero as pull-

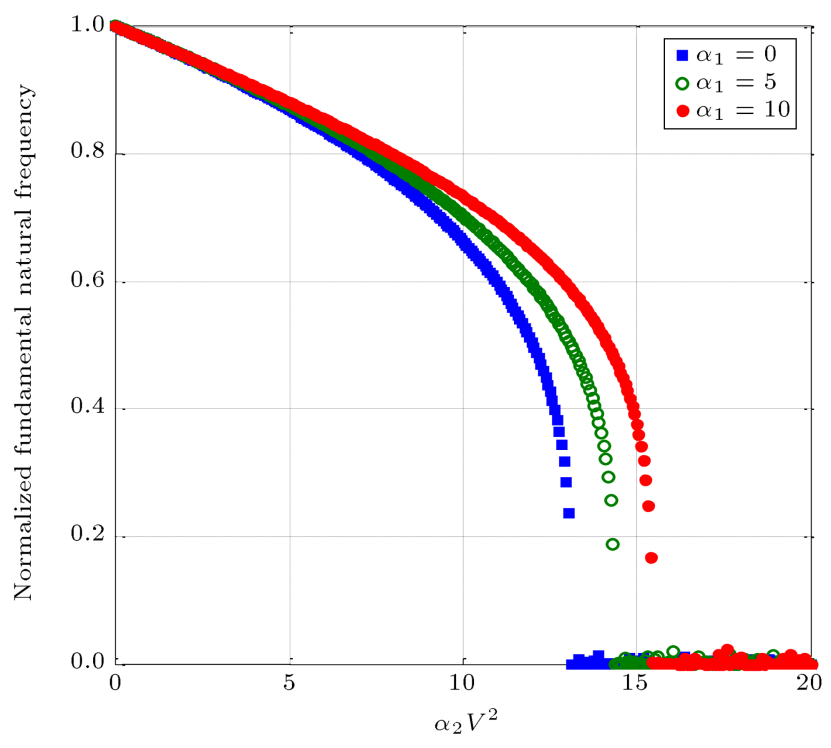

Figure 15. Fundamental natural frequency of deflected clamped circular plate versus applied voltage for various values of $\alpha_{1}$.

in develops. For high values of $\alpha_{1}$, the fundamental natural frequency first increases with electrostatic load due to strain hardening, and then decreases and approaches zero. In clamped-circular plate, the stiffness monotony decreases with an increase in applied voltage and, accordingly, natural frequency decreases. For annular plate with smaller inner radius, the condition is the same, while, for higher values of inner radius, the fundamental frequency of plate increases at first since there is limitation on the transverse displacement of the plate; finally, at higher values of applied pull-in voltage, zero frequency occurs. Due to limitation on the transverse displacement of annular plate with a bigger inner radius, when the value of $\alpha_{1}$ is increased, there is a pronounced hardening effect for higher values of $\alpha_{1}$ and $\beta$, and natural frequency increases accordingly in some frequency ranges of applied voltage.

\section{Conclusion}

In this paper, the static pull-in behavior and natural frequency of vibration for electrostatically actuated circular and annular plates were investigated. It was found that increasing the tensile radial force increases the pull-in voltage, and increasing the compressive radial force decreases it. Moreover, results showed that increasing the inner radius of annular plates causes an increase in the static pull-in voltage; by increasing geometric nonlinearity, pull-in voltage develops in bigger voltage. Subsequently, the effect of applied voltage on fundamental natural frequency of deflected plates was investigated. Results showed that by increasing applied voltage, the fundamental natural frequency decreases and approaches zero as pull-in voltage develops. The 


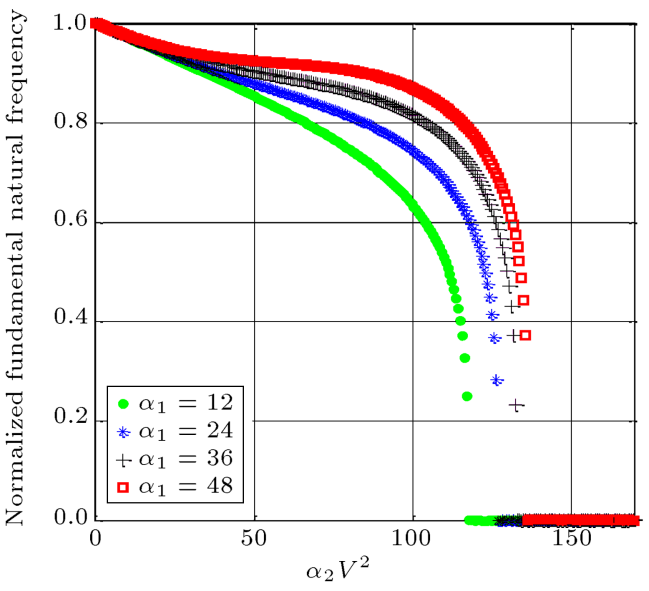

(a)

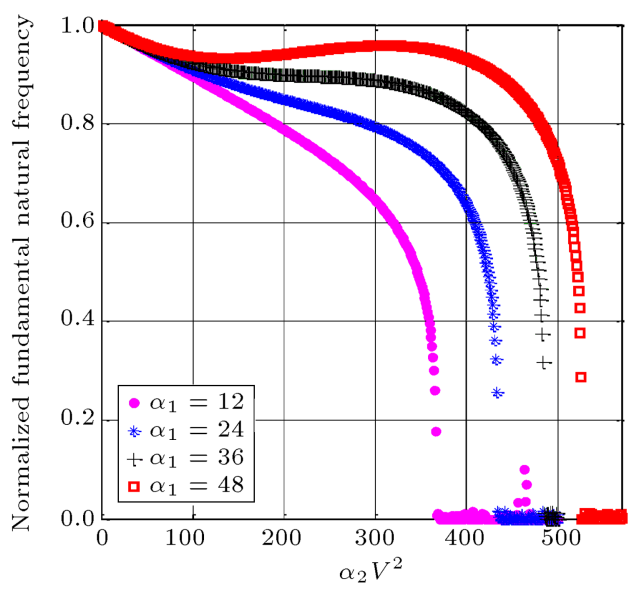

(b)

Figure 16. Fundamental natural frequency of deflected clamped-clamped annular plate with various values of $\alpha_{1}$ : (a) $\beta=0.1$, and (b) $\beta=0.3$.

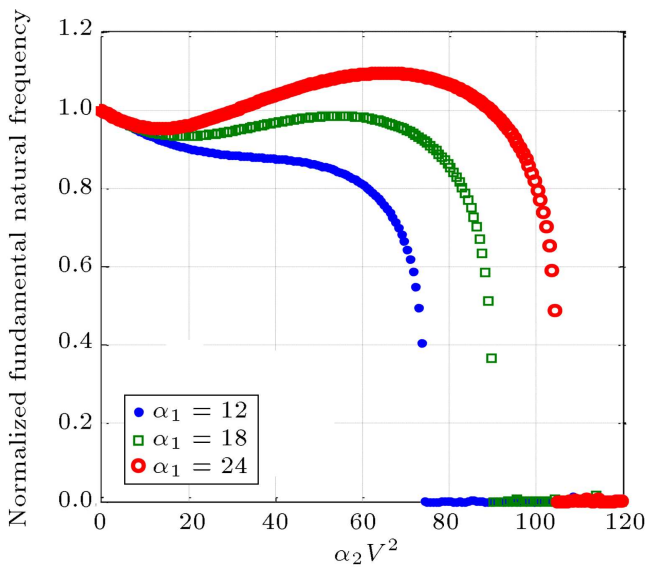

(a)

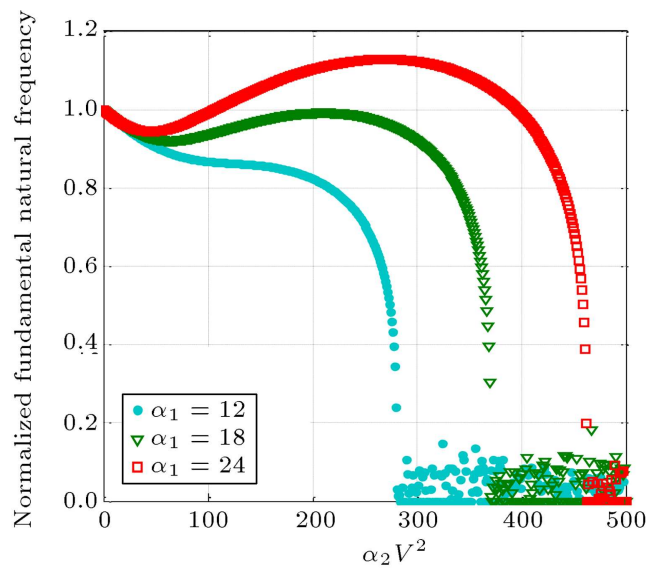

(b)

Figure 17. Fundamental natural frequency of deflected clamped-simply supported annular plate for various $\alpha_{1}$ : (a) $\beta=0.1$, and $(\mathrm{b}) \beta=0.3$.

obtained results show that variations of different parameters have great influence on pull-in voltage and small influence on deflection at pulling point. This means that deflection at the pull-in condition is nearly the same for different parameters of a plate and is more influenced by the boundary condition with respect to other parameters of system.

\section{NOMENCLATURE}

$R$

Radius of the plate

$h$

Thickness of the plate

d Gap between two electrodes

$b \quad$ Radius of rigid core

$h_{c} \quad$ Thickness of rigid core

$M_{\text {core }} \quad$ Mass of rigid core

$\varepsilon \quad$ The dielectric constant of the gap medium $t$

E

$w$

$u, v$

$I_{2}$

$\bar{w}$

$D$

$\alpha_{1}$

$I_{d}$ $N_{r r}, N_{\theta \theta}$,

$N_{r \theta} \quad$ In-plane forces

$T \quad$ The kinetic energy

$T_{\text {core }} \quad$ The kinetic energy of rigid core

$\nu \quad$ Poisson's ratio

\section{Young's modulus}

Transverse displacement of neutral axes in the $z$ direction In-plane displacements Rotary inertia Dimensionless transverse displacement The flexural rigidity of plate

Geometric structural nonlinearity parameter

Related to the radius of gyration 


$\begin{array}{ll}\rho & \text { Mass density of the plate } \\ \rho_{c} & \text { Mass density of rigid core } \\ G & \text { Shear modulus } \\ \delta & \text { Variational operator } \\ P_{r} & \text { Radial force per length } \\ f_{e s} & \text { Electrostatic force per unit area } \\ c & \text { Damping coefficient } \\ V & \text { Applied voltage } \\ u_{r}, u_{\theta}, u_{z} & \text { Displacements in the } r, \theta, \text { and } z \\ I_{0} & \text { directions } \\ \bar{V} & \text { Mass per unit area } \\ \bar{r} & \text { Dimensionless voltage } \\ \bar{t} & \text { Dimensionless radius } \\ \alpha_{2} & \text { Dimensionless time } \\ \bar{P}_{r} & \text { Electrostatic force parameter } \\ M_{r r}, M_{\theta \theta}, & \text { Dimensionless radial load } \\ M_{r \theta} & \text { Out of plane moments } \\ I I & \text { The strain energy } \\ \Omega_{0} & \text { Domain of the plate }\end{array}$

\section{References}

1. Nathanson, H.C., Newell, W.E., Wickstrom, R.A., and Davis, J.R. "The resonant gate transistor", IEEE Transactions on Electron Devices, 14, pp. 117-133 (1967).

2. Taylor, G. "The coalescence of closely spaced drops when they are at different electric potentials", Proceedings of the Royal Society of London, A: Mathematical, Physical and Engineering Sciences, 306, pp. 423-434 (1968).

3. Chao, P.C., Chiu, C., and Liu, T.-H. "DC dynamic pull-in predictions for a generalized clamped-clamped micro-beam based on a continuous model and bifurcation analysis", Journal of Micromechanics and Microengineering, 18, p. 115008 (2008).

4. Krylov, S. "Lyapunov exponents as a criterion for the dynamic pull-in instability of electrostatically actuated microstructures", International Journal of Non-Linear Mechanics, 42, pp. 626-642 (2007).

5. Hung, E.S. and Senturia, S.D. "Extending the travel range of analog-tuned electrostatic actuators", Journal of Microelectromechanical Systems, 8, pp. 497-505 (1999).

6. Bochobza-Degani, O. and Nemirovsky, Y. "Modeling the pull-in parameters of electrostatic actuators with a novel lumped two degrees of freedom pull-in model", Sensors and Actuators A: Physical, 97, pp. 569-578 (2002).
7. Bochobza-Degani, O. and Nemirovsky, Y. "Experimental verification of a design methodology for torsion actuators based on a rapid pull-in solver", Microelectromechanical Systems, Journal of, 13, pp. 121-130 (2004).

8. Madinei, H., Rezazadeh, G., and Azizi, S. "Stability and bifurcation analysis of an asymmetrically electrostatically actuated microbeam", Journal of Computational and Nonlinear Dynamics, 10, p. 021002 (1-8) (2015).

9. Hsu, M. "Deflection analysis of electrostatic microactuators using the differential quadrature method", Tamkang Journal of Science and Engineering, 9, pp. 97-106 (2006).

10. Batra, R.C., Porfiri, M., and Spinello, D. "Electromechanical model of electrically actuated narrow microbeams", Journal of Microelectromechanical Systems, 15, pp. 1175-1189 (2006).

11. Huang, Y.-T., Chen, H.-L., and Hsu, W. "An analytical model for calculating the pull-in voltage of micro cantilever beams subjected to tilted and curled effects", Microelectronic Engineering, 125, pp. 73-77 (2014).

12. Beni, Y.T. and M. Heidari, "Numerical study on pull-in instability analysis of geometrically nonlinear Euler-Bernoulli microbeam based on modified couple stress theory", International Journal of Engineering and Applied Sciences, 4, pp. 41-53 (2012).

13. Zhang, Y. and Zhao, Y.-P. "Numerical and analytical study on the pull-in instability of micro-structure under electrostatic loading", Sensors and Actuators A: Physical, 127, pp. 366-380 (2006).

14. Chowdhury, S., Ahmadi, M., and Miller, W. "A comparison of pull-in voltage calculation methods for MEMS-based electrostatic actuator design", 1st International Conference on Sensing Technology, November, Palmerston North, New Zealand, pp. 21-23 (2005)

15. Mojahedi, M. and Rahaeifard, M. "Static deflection and pull-in instability of the electrostatically actuated bilayer microcantilever beams", International Journal of Applied Mechanics, 7, pp. 1-15 (2015).

16. Baghania, M., Asgarshamsib, A., and Goharkhaha, M. "Analytical solution for large amplitude vibrations of microbeams actuated by an electro-static force", Scientia Iranica, Transactions B, Mechanical Engineering, 20, pp. 1499-1507 (2013).

17. Rahaeifard, M. and Ahmadian, M. "On pull-in instabilities of microcantilevers", International Journal of Engineering Science, 87, pp. 23-31 (2015).

18. Gholami, R., Ansari, R., and Rouhi, H. "Studying the effects of small scale and Casimir force on the non-linear pull-in instability and vibrations of FGM 
microswitches under electrostatic actuation", International Journal of Non-Linear Mechanics, 77, pp. 193207 (2015).

19. Xiao, Y., Wang, B., and Zhou, S. "Pull-in voltage analysis of electrostatically actuated MEMS with piezoelectric layers: A size-dependent model", Mechanics Research Communications, 66, pp. 7-14 (2015).

20. Srinivas, D. "Electromechanical dynamics of simplysupported micro-plates", International Journal of Computational Engineering Research, 2, pp. 1388-1395 (2012).

21. Mukherjee, S., Bao, Z., Roman, M., and Aubry, N. "Nonlinear mechanics of MEMS plates with a total Lagrangian approach", Computers \& structures, 83, pp. $758-768$ (2005).

22. Zhao, X., Abdel-Rahman, E.M., and Nayfeh, A.H. "A reduced-order model for electrically actuated microplates", Journal of Micromechanics and Microengineering, 14, pp. 900-906 (2004).

23. Zand, M.M., Rashidian, B., and Ahmadian, M. "Contact time study of electrostatically actuated microsystems", Scientia Iranica, Transactions B, Mechanical Engineering, 17, pp. 346-357 (2010).

24. Wang, K., Kitamura, T., and Wang, B. "Nonlinear pull-in instability and free vibration of micro/nanoscale plates with surface energy-A modified couple stress theory model", International Journal of Mechanical Sciences, 99, pp. 288-296 (2015).

25. Saif, M., Alaca, B.E., and Sehitoglu, H. "Analytical modeling of electrostatic membrane actuator for micro pumps", Journal of Microelectromechanical Systems, 8, pp. 335-345 (1999).

26. Wang, Y.G., Lin, W.H., Li, X.M., and Feng, Z.J. "Bending and vibration of an electrostatically actuated circular microplate in presence of Casimir force", Applied Mathematical Modelling, 35, pp. 2348-2357 (2011).

27. Soleymani, P., Sadeghian, H., Tahmasebi, A., and Rezazadeh, Gh. "Pull-in instability investigation of circular micro pump subjected to nonlinear electrostatic force", Sensors \& Transducers, 69, pp. 622-628 (2006).

28. Nayfeh, A.H., Younis, M.I., and Abdel-Rahman, E.M. "Reduced-order models for MEMS applications", Nonlinear Dynamics, 41, pp. 211-236 (2005).

29. Reddy, J.N., Theory and Analysis of Elastic Plates and Shells, CRC Press (2006).

30. Magrab, E.B., Vibrations of Elastic Systems: With Applications to MEMS and NEMS, Springer Science \& Business Media, 184 (2012).
31. Timoshenko, S.P. and Gere, J.M., Theory of Elastic Stability, Courier Corporation (2009).

32. Pelesko, J.A. and Bernstein, D.H., Modeling Mems and Nems, CRC press (2002).

33. Rao, S.S., Vibration of Continuous Systems, John Wiley \& Sons (2007).

34. Seydel, R., Practical Bifurcation and Stability Analysis, Springer Science \& Business Media, 5 (2009).

35. Galâan-Vioque, J., Krauskopf, B., and Osinga, H.M., Numerical Continuation Methods for Dynamical Systems: Path Following and Boundary Value Problems, Springer (2007).

36. Nayfeh, A.H. and Balachandran, B., Applied Nonlinear Dynamics: Analytical, Computational and Experimental Methods, John Wiley \& Sons (2008).

37. Parseh, M., Dardel, M., and Ghasemi, M.H. "Investigating the robustness of nonlinear energy sink in steady state dynamics of linear beams with different boundary conditions", Communications in Nonlinear Science and Numerical Simulation, 29, pp. 50-71 (2015).

38. Vogl, G.W. "Nonlinear dynamics of circular plates under electrical loadings for capacitive micromachined ultrasonic transducers (CMUT)", PhD Dissertation Blacksburg, VirginiaTech (2006).

\section{Appendix A}

In this appendix, by employing extended Hamilton's principle, Eq. (12) will be obtained. Extended Hamilton's principle is given as follows [29]:

$$
\int_{t_{1}}^{t_{2}}(\delta \Pi-\delta W-\delta T) d t=0 .
$$

By substituting Eqs. (4)-(11) into Eq. (A.1), the extended Hamilton's principle can be written as:

$$
\begin{array}{r}
\int_{t_{1}}^{t_{2}} \iint_{\Omega_{0}} \int_{-h / 2}^{h / 2}\left(\sigma_{r r} \delta \epsilon_{r r}+\sigma_{\theta \theta} \delta \epsilon_{\theta \theta}+\sigma_{r \theta} \delta \gamma_{r \theta}\right) d z r d r d \theta d t \\
-\int_{t_{1}} \iint_{\Omega_{0}} \int_{-h / 2}^{h / 2} \rho\left(\dot{u}_{r} \delta \dot{u}_{r}+\dot{u}_{\theta} \delta \dot{u}_{\theta}+\dot{u}_{z} \delta \dot{u}_{z}\right) d z r d r d \theta d t \\
-\int_{t_{1}}^{t_{2}} \frac{1}{2} M_{\text {core }}\left(\left.\frac{\partial w}{\partial t}\right|_{r=b}\right)^{2} d t-\int_{t_{1}}^{t_{2}} \iint_{\Omega_{0}} f_{e s} \delta w r d r d \theta d t \\
-\int_{t_{1}}^{t_{2}} \int_{\Omega_{0}} \int_{t_{2}} \delta\left(P_{r} \epsilon_{r r}\right) r d r d \theta d t+\delta \int_{t_{1}}^{t_{2}} \int_{\Omega_{0}} c \frac{\partial w}{\partial t} w r d r d \theta d t=0 . \\
\text { (A.2) }
\end{array}
$$

By using terms defined in Eq. (14), the variations of the kinetics and strain energy can be written as follows: 


$$
\begin{aligned}
& \int_{t_{1}}^{t_{2}} \delta \Pi d t=\int_{t_{1}}^{t_{2}} \iint_{\Omega_{0}} \int_{-h / 2}^{h / 2}\left(\sigma_{r r} \delta \epsilon_{r r}+\sigma_{\theta \theta} \delta \epsilon_{\theta \theta}+\sigma_{r \theta} \delta \gamma_{r \theta}\right) \\
& d z r d r d \theta d t=\int_{t_{1}}^{t_{2}} \iint_{\Omega_{0}}\left\{\int _ { - h / 2 } ^ { h / 2 } \sigma _ { r r } \left[\frac{\partial w}{\partial r} \frac{\partial \delta w}{\partial r}\right.\right. \\
& \left.-z \frac{\partial^{2} \delta w}{\partial r^{2}}\right] d z+\int_{-h / 2}^{h / 2} \sigma_{\theta \theta}\left[\frac{1}{r^{2}} \frac{\partial w}{\partial \theta} \frac{\partial \delta w}{\partial \theta}\right. \\
& \left.-\frac{z}{r}\left(\frac{\partial \delta w}{\partial r}+\frac{1}{r} \frac{\partial^{2} \delta w}{\partial \theta^{2}}\right)\right] d z \\
& +\int_{-h / 2}^{h / 2} \sigma_{r \theta}\left[\frac{1}{r} \frac{\partial \delta w}{\partial r} \frac{\partial w}{\partial \theta}+\frac{1}{r} \frac{\partial w}{\partial r} \frac{\partial \delta w}{\partial \theta}\right. \\
& \left.\left.-2 \frac{z}{r}\left(\frac{\partial^{2} \delta w}{\partial r \partial \theta}-\frac{1}{r} \frac{\partial \delta w}{\partial \theta}\right)\right] d z\right\} r d r d \theta d t \\
& =\int_{t_{1}}^{t_{2}} \iint_{\Omega_{0}}\left\{N_{r r}\left(\frac{\partial w}{\partial r} \frac{\partial \delta w}{\partial r}\right)-M_{r r} \frac{\partial^{2} \delta w}{\partial r^{2}}\right. \\
& +N_{\theta \theta}\left(\frac{1}{r^{2}} \frac{\partial w}{\partial \theta} \frac{\partial \delta w}{\partial \theta}\right) \\
& -\frac{M_{\theta \theta}}{r}\left(\frac{\partial \delta w}{\partial r}+\frac{1}{r} \frac{\partial^{2} \delta w}{\partial \theta^{2}}\right) \\
& +N_{r \theta}\left(\frac{1}{r} \frac{\partial \delta w}{\partial r} \frac{\partial w}{\partial \theta}+\frac{1}{r} \frac{\partial w}{\partial r} \frac{\partial \delta w}{\partial \theta}\right) \\
& \left.-2 \frac{M_{r \theta}}{r}\left(\frac{\partial^{2} \delta w}{\partial r \partial \theta}-\frac{1}{r} \frac{\partial \delta w}{\partial \theta}\right)\right\} r d r d \theta d t,(\mathrm{~A} .3)
\end{aligned}
$$$$
\int_{t_{1}}^{t_{2}} \delta T d t=\int_{t_{1}}^{t_{2}} \iint_{\Omega_{0}} \int_{-h / 2}^{h / 2} \rho\left(\dot{u}_{r} \delta \dot{u}_{r}+\dot{u}_{\theta} \delta \dot{u}_{\theta}+\dot{u}_{z} \delta \dot{u}_{z}\right)
$$

$$
\begin{aligned}
& d z r d r d \theta d t+\int_{t_{1}}^{t_{2}} \frac{1}{2} M_{\text {core }}\left(\left.\frac{\partial w}{\partial t}\right|_{r=b}\right)^{2} d t \\
& =\int_{t_{1}}^{t_{2}} \iint_{\Omega_{0}} \int_{-h / 2}^{h / 2} \rho\left[\left(-z \frac{\partial^{2} w}{\partial r \partial t}\right)\left(-z \frac{\partial^{2} \delta w}{\partial r \partial t}\right)\right. \\
& +\left(-\frac{z}{r} \frac{\partial^{2} w}{\partial t \partial \theta}\right)\left(-\frac{z}{r} \frac{\partial^{2} \delta w}{\partial t \partial \theta}\right)
\end{aligned}
$$

$$
\begin{aligned}
& +\dot{w} \delta \dot{w}] d z r d r d \theta d t+\int_{t_{1}}^{t_{2}} \frac{1}{2} M_{\text {core }}\left(\left.\frac{\partial w}{\partial t}\right|_{r=b}\right)^{2} d t \\
& =\int_{t_{1}} \int_{\Omega_{0}}^{t_{2}}\left(\int_{2} \frac{\partial^{2} w}{\partial r \partial t} \frac{\partial^{2} \delta w}{\partial r \partial t}+\frac{I_{2}}{r^{2}} \frac{\partial^{2} w}{\partial t \partial \theta} \frac{\partial^{2} \delta w}{\partial t \partial \theta}\right. \\
& \left.+I_{0} \dot{w} \delta \dot{w}\right) r d r d \theta d t+\int_{t_{1}}^{t_{2}} \frac{1}{2} M_{\text {core }}\left(\left.\frac{\partial w}{\partial t}\right|_{r=b}\right)^{2} d t
\end{aligned}
$$

By substituting Eqs. (A.3) and (A.4) into Eq. (A.2), we obtain:

$$
\begin{aligned}
& \int_{t_{1}}^{t_{2}} \iint_{\Omega_{0}}\left[N_{r r}\left(\frac{\partial w}{\partial r} \frac{\partial \delta w}{\partial r}\right)+N_{\theta \theta}\left(\frac{1}{r^{2}} \frac{\partial w}{\partial \theta} \frac{\partial \delta w}{\partial \theta}\right)\right. \\
& +N_{r \theta}\left(\frac{1}{r} \frac{\partial \delta w}{\partial r} \frac{\partial w}{\partial \theta}+\frac{1}{r} \frac{\partial w}{\partial r} \frac{\partial \delta w}{\partial \theta}\right)-M_{r r} \frac{\partial^{2} \delta w}{\partial r^{2}} \\
& -\frac{M_{\theta \theta}}{r}\left(\frac{\partial \delta w}{\partial r}+\frac{1}{r} \frac{\partial^{2} \delta w}{\partial \theta^{2}}\right)-2 \frac{M_{r \theta}}{r}\left(\frac{\partial^{2} \delta w}{\partial r \partial \theta}\right. \\
& \left.\left.-\frac{1}{r} \frac{\partial \delta w}{\partial \theta}\right)\right] r d r d \theta d t \int_{t_{1}}^{t_{2}} \iint_{\Omega_{0}}\left[I_{0}(\dot{w} \delta \dot{w})\right. \\
& \left.+I_{2}\left(\frac{\partial^{2} w}{\partial r \partial t} \frac{\partial^{2} \delta w}{\partial r \partial t}+\frac{1}{r^{2}} \frac{\partial^{2} w}{\partial t \partial \theta} \frac{\partial^{2} \delta w}{\partial t \partial \theta}\right)\right] r d r d \theta d t \\
& +\int_{t_{1}}^{t_{2}} \int_{\Omega_{0}} \int_{f_{e s}} \delta w r d r d \theta d t-\int_{t_{1}}^{t_{2}} \iint_{\Omega_{0}} \delta\left(P_{r} \epsilon_{r r}\right) r d r d \theta d t \\
& +\int_{t_{1}}^{t_{2}} \int_{\Omega_{0}} c \frac{\partial w}{\partial t} w r d r d \theta d t=0 .
\end{aligned}
$$

By integrating Eq. (A.5) by parts in space and time to relive $\delta w$ from any differentiations, it is found that each individual term simplifies as follows:

$$
\begin{aligned}
& \iint_{\Omega_{0}} N_{r r} \frac{\partial w}{\partial r} \frac{\partial \delta w}{\partial r} r d r d \theta \\
& \quad=\left.\int_{\theta_{1}}^{\theta_{2}} r N_{r r} \frac{\partial w}{\partial r} \delta w\right|_{r_{1}} ^{r_{2}} d \theta-\iint_{\Omega_{0}} \frac{\partial}{\partial r}\left(r N_{r r} \frac{\partial w}{\partial r}\right) \delta w d r d \theta \\
& (\mathrm{A} .6)
\end{aligned}
$$




$$
\begin{aligned}
& \iint_{\Omega_{0}} N_{r \theta} \frac{1}{r} \frac{\partial w}{\partial \theta} \frac{\partial \delta w}{\partial r} r d r d \theta \\
& =\left.\int_{\theta_{1}}^{\theta_{2}} N_{r \theta} \frac{\partial w}{\partial \theta} \delta w\right|_{r_{1}} ^{r_{2}} d \theta-\iint_{\Omega_{0}} \frac{\partial}{\partial r}\left(N_{r \theta} \frac{\partial w}{\partial \theta}\right) \begin{array}{r}
\delta w d r d \theta, \\
(\mathrm{A} .8)
\end{array} \\
& \iint_{\Omega_{0}} N_{r \theta} \frac{1}{r} \frac{\partial w}{\partial r} \frac{\partial \delta w}{\partial \theta} r d r d \theta \\
& =\left.\int_{r_{1}}^{r_{2}} N_{r \theta} \frac{\partial w}{\partial r} \delta w\right|_{\theta_{1}} ^{\theta_{2}} d r-\iint_{\Omega_{0}} \frac{\partial}{\partial \theta}\left(N_{r \theta} \frac{\partial w}{\partial r}\right) \begin{array}{r}
\delta w d r d \theta, \\
(\mathrm{A} .9)
\end{array} \\
& -\iint_{\Omega_{0}} M_{r r} \frac{\partial^{2} \delta w}{\partial r^{2}} r d r d \theta=-\left.\int_{\theta_{1}}^{\theta_{2}} r M_{r r} \delta\left(\frac{\partial w}{\partial r}\right)\right|_{r_{1}} ^{r_{2}} d \theta \\
& +\left.\int_{\theta_{1}}^{\theta_{2}} \frac{\partial\left(r M_{r r}\right)}{\partial r} \delta w\right|_{r_{1}} ^{r_{2}} d \theta-\iint_{\Omega_{0}} \frac{\partial^{2}\left(r M_{r r}\right)}{\partial r^{2}} \delta w d r d \theta \\
& -\iint_{\Omega_{0}} \frac{M_{\theta \theta}}{r} \frac{\partial \delta w}{\partial r} r d r d \theta=-\left.\int_{\theta_{1}}^{\theta_{2}} M_{\theta \theta} \delta w\right|_{r_{1}} ^{r_{2}} d \theta \\
& +\iint_{\Omega_{0}} \frac{\partial M_{\theta \theta}}{\partial r} \delta w d r d \theta \\
& -\iint_{\Omega_{0}} \frac{M_{\theta \theta}}{r} \frac{1}{r} \frac{\partial^{2} \delta w}{\partial \theta^{2}} r d r d \theta= \\
& -\left.\int_{r_{1}}^{r_{2}} \frac{M_{\theta \theta}}{r} \delta\left(\frac{\partial w}{\partial \theta}\right)\right|_{\theta_{1}} ^{\theta_{2}} d r+\left.\int_{r_{1}}^{r_{2}} \frac{\partial}{\partial \theta}\left(\frac{M_{\theta \theta}}{r}\right) \delta w\right|_{\theta_{1}} ^{\theta_{2}} d r \\
& -\iint_{\Omega_{0}} \frac{\partial^{2}}{\partial \theta^{2}}\left(\frac{M_{\theta \theta}}{r}\right) \delta w d r d \theta
\end{aligned}
$$$$
-2 \iint_{\Omega_{0}} M_{r \theta} \frac{1}{r}\left(\frac{\partial^{2} \delta w}{\partial r \partial \theta}-\frac{1}{r} \frac{\partial \delta w}{\partial \theta}\right) r d r d \theta=
$$$$
-\left.2 \int_{r_{1}}^{r_{2}} M_{r \theta} \delta\left(\frac{\partial w}{\partial r}\right)\right|_{\theta_{1}} ^{\theta_{2}} d r
$$$$
+\left.\left.2 \frac{\partial M_{r \theta}}{\partial \theta} \delta w\right|_{\theta_{1}} ^{\theta_{2}}\right|_{r_{1}} ^{r_{2}}-2 \iint_{\Omega_{0}} \frac{\partial^{2} M_{r \theta}}{\partial r \partial \theta} \delta w d r d \theta
$$$$
+\left.2 \int_{r_{1}}^{r_{2}} \frac{M_{r \theta}}{r} \delta w\right|_{\theta_{1}} ^{\theta_{2}} d r-2 \iint_{\Omega_{0}} \frac{1}{r} \frac{\partial M_{r \theta}}{\partial \theta} \delta w d r d \theta
$$

$$
\begin{aligned}
-2 \iint_{\Omega_{0}} M_{r \theta} \frac{1}{r} \frac{\partial^{2} \delta w}{\partial r \partial \theta} r d r d \theta= \\
\quad-\iint_{\Omega_{0}} M_{r \theta} \frac{\partial^{2} \delta w}{\partial r \partial \theta} d r d \theta-\iint_{\Omega_{0}} M_{r \theta} \frac{\partial^{2} \delta w}{\partial r \partial \theta} d r d \theta \\
\quad=-\left.\left.2 M_{r \theta} \delta\left(\frac{\partial w}{\partial r}\right)\right|_{\theta_{1}} ^{\theta_{2}}\right|_{r_{1}} ^{r_{2}}+\left.\left.\int_{r_{1}}^{r_{2}} \frac{\partial M_{r \theta}}{\partial r}\right|_{\theta_{1}} ^{\theta_{2}} \delta w\right|_{\theta_{1}} ^{\theta_{2}} d r \\
+\left.\left.\int_{\theta_{1}}^{\theta_{2}} \frac{\partial M_{r \theta}}{\partial \theta}\right|_{r_{1}} ^{r_{2}} \delta w\right|_{r_{1}} ^{r_{2}} d \theta+\left.\int_{\theta_{1}}^{\theta_{2}} \frac{\partial M_{r \theta}}{\partial \theta} \delta w\right|_{r_{1}} ^{r_{2}} d \theta \\
\quad+\left.\int_{r_{1}}^{r_{2}} \frac{\partial M_{r \theta}}{\partial r} \delta w\right|_{\theta_{1}} ^{\theta_{2}} d r-\iint_{\Omega_{0}} 2 \frac{\partial^{2} M_{r \theta}}{\partial r \partial \theta} \delta w d r d \theta, \quad \text { (A.14) }
\end{aligned}
$$$$
2 \iint_{\Omega_{0}} M_{r \theta} \frac{1}{r} \frac{\partial \delta w}{\partial \theta} d r d \theta
$$$$
=\left.\int_{r_{1}}^{r_{2}} \frac{2}{r} M_{r \theta} \delta w\right|_{\theta_{1}} ^{\theta_{2}} d r-2 \iint_{\Omega_{0}} \frac{1}{r} \frac{\partial M_{r \theta}}{\partial \theta} \delta w d r d \theta
$$

$$
-\int_{t_{1}}^{t_{2}} \iint_{\Omega_{0}} I_{0}(\dot{w} \delta \dot{w}) r d r d \theta d t=\int_{t_{1}}^{t_{2}} \iint_{\Omega_{0}} r I_{0}(\ddot{w} \delta w) d r d \theta d t
$$$$
-\int_{t_{1}}^{t_{2}} \int_{\Omega_{0}} \int_{2}\left(\frac{\partial^{2} w}{\partial r \partial t} \frac{\partial^{2} \delta w}{\partial r \partial t}+\frac{1}{r^{2}} \frac{\partial^{2} w}{\partial t \partial \theta} \frac{\partial^{2} \delta w}{\partial t \partial \theta}\right) r d r d \theta d t
$$

$$
\begin{aligned}
& =\int_{t_{1}}^{t_{2}}\left[\left.\int_{\theta_{1}}^{\theta_{2}} r I_{2_{2}} \frac{\partial^{3} w}{\partial r \partial t^{2}} \delta w\right|_{r_{1}} ^{r_{2}} d \theta+\left.\int_{r_{1}}^{r_{2}} \frac{1}{r} I_{2} \frac{\partial^{3} w}{\partial t^{2} \partial \theta} \delta w\right|_{\theta_{1}} ^{\theta_{2}} d r\right. \\
& \left.-\iint_{\Omega_{0}} I_{2} \frac{\partial^{2}}{\partial t^{2}}\left[\frac{\partial}{\partial r}\left(r \frac{\partial w}{\partial r}\right)+\frac{1}{r} \frac{\partial^{2} w}{\partial \theta^{2}}\right] \delta w d r d \theta d t\right] .
\end{aligned}
$$

By substituting Eqs. (A.6)-(A.17) into Eq. (A.5) and setting each of the coefficients of in the area integrand equal to zero, the final form of equation of motion and boundary conditions will be obtained as Eqs. (12) and (13).

\section{Biographies}

Masoumeh Khorshidi Paji received BS and MS degrees in Engineering Mechanics from Babol Noshirvani University of Technology, Iran in 2012 and 2015, respectively. Her research interests include MEMS and NEMS, nonlinear vibrations and dynamics, smart structures, and energy harvesting. 
Morteza Dardel received a PhD degree from Amirkabir University, Iran in Solid Mechanics in 2009, and is currently an Assistant Professor in the Faculty of Mechanical Engineering at Babol Noshirvani University of Technology, Iran. His research interests are aeroelasticity, nonlinear dynamics, vibration, and control of continuous systems and smart structures.

Mohammad Hadi Pashaei received a $\mathrm{PhD}$ degree in Space Structures from the University of Surrey, UK in 2004, and is currently an Assistant Professor in the Faculty of Mechanical Engineering at Babol Noshirvani University of Technology, Iran. His research interests include structural dynamics, damping in structures, and vibrational systems analysis.
Reza Akbari Alashti was born in Iran in 1963. He received a $\mathrm{PhD}$ degree in Mechanical Engineering, Applied Design, from Tarbiat Modares University, Tehran, Iran in 2006, focused on the limit load analysis of cylindrical shells with opening under combined loading using constrained minimization techniques. Since 2006, he has been a faculty member in the Mechanical Engineering Department of Babol Noshirvani University of Technology, Iran. Dr. Akbari Alashti is a member of the Iranian Society of Mechanical Engineering, European Society of Mechanical Engineers, and the Iranian Association of Naval Architecture and Marine Engineers. His research interests include stress analysis in shells and plates, nonlinear analysis of structures, limit load, and damage analysis. 